\title{
Flow Equation Approach to Periodically Driven Quantum Systems
}

\author{
Michael Vogl, ${ }^{* \dagger}$ Pontus Laurell, , and Aaron D. Barr \\ Department of Physics, The University of Texas at Austin, Austin, Texas 78712, USA \\ Gregory A. Fiete \\ Department of Physics, Northeastern University, Boston, Massachusetts 02115, USA; \\ Department of Physics, The University of Texas at Austin, Austin, Texas 78712, USA \\ and Department of Physics, Massachusetts Institute of Technology, \\ Cambridge, Massachusetts 02139, USA
}

(Received 8 August 2018; revised manuscript received 19 March 2019; published 23 May 2019)

\begin{abstract}
We present a theoretical method to generate a highly accurate time-independent Hamiltonian governing the finite-time behavior of a time-periodic system. The method exploits infinitesimal unitary transformation steps, from which renormalization-group-like flow equations are derived to produce the effective Hamiltonian. Our tractable method has a range of validity reaching into frequency-and drive strength-regimes that are usually inaccessible via high-frequency $\omega$ expansions in the parameter $h / \omega$, where $h$ is the upper limit for the strength of local interactions. We demonstrate exact properties of our approach on a simple toy model and test an approximate version of it on both interacting and noninteracting many-body Hamiltonians, where it offers an improvement over the more well-known Magnus expansion and other high-frequency expansions. For the interacting models, we compare our approximate results to those found via exact diagonalization. While the approximation generally performs better globally than other highfrequency approximations, the improvement is especially pronounced in the regime of lower frequencies and strong external driving. This regime is of special interest because of its proximity to the resonant regime where the effect of a periodic drive is the most dramatic. Our results open a new route towards identifying novel nonequilibrium regimes and behaviors in driven quantum many-particle systems.
\end{abstract}

DOI: 10.1103/PhysRevX.9.021037

\section{INTRODUCTION}

Recent years have seen rapid progress in our understanding of dynamics and nonequilibrium phenomena in quantum systems $[1,2]$. This has been a result of experimental advances in the ability to control cold atom [2-4] and condensed matter systems [5-7], by developments in timeresolved laser techniques [8,9], and by the fact that stepping into the time domain opens up new ways of ultrafast control of material properties $[5,10,11]$ and access to different phases of matter. These include photoinduced superconductivity [12,13], hidden orders [14], and metastable states [15], but also entirely novel phases, such as time crystals $[16,17]$ and nonequilibrium topological phases $[18,19]$.

\footnotetext{
*These authors contributed equally to this work.

Corresponding author. ssss133@googlemail.com

Published by the American Physical Society under the terms of the Creative Commons Attribution 4.0 International license. Further distribution of this work must maintain attribution to the author(s) and the published article's title, journal citation, and DOI.
}

Subject Areas: Condensed Matter Physics, Quantum Physics
In particular, there has been growing interest in periodically driven (or Floquet) [20,21] many-body systems, which can bear a close resemblance to equilibrium systems [22]. The Floquet systems come in three established thermodynamic classes: integrable [23-25], many-body localized (MBL) [19,26,27], and generic interacting ones [28]. The first two classes can avoid thermalization, allowing for a notion of a Floquet phase of matter at long stroboscopic times $t=n T$, where $T$ is the period of the Hamiltonian, $H(t+T)=H(t)$, and $n$ is an integer. The physics of these phases is captured by an effective, timeindependent Floquet Hamiltonian $H_{F}$, given via the timeevolution operator over one period $U(T)=\exp \left(-i H_{F} T\right)$.

In noninteracting systems, $H_{F}$ can be used to dynamically engineer interesting and topological band structures [29-38], most notably Floquet topological insulators [39-44]. Our main interest, however, is in interacting systems where $H_{F}$ can be engineered to drive phase transitions $[45,46]$ or, in the case of Floquet-MBL systems, realize new phases without equilibrium analogs $[18,19$, 47-49]. Clean interacting Floquet systems are the least studied of the three classes, perhaps because they were 
expected to heat up to a featureless state with infinite effective temperature [50,51]. However, it was recently theoretically discovered that under very general conditions they may remain in a prethermal state until exponentially long times $\tau^{\star}$ [52-56], which has been verified numerically in several models $[57,58]$.

The existence of a prethermal regime is important because realistic systems usually contain integrabilitybreaking perturbations that support it and because the thermalization (or more specifically, the energy absorption) time $\tau^{\star}$ can correspond to experimentally accessible timescales. The existence of such a regime also implies that there is interesting physics to be found at intermediate times $0<t<\tau^{\star}$ [52,59], where one may use time-dependent perturbations to drive dynamical phase transitions [60-63], control interactions $[64,65]$, or engineer phase transitions and topological phases [66-70].

To understand the properties of a system in the prethermal regime, it is convenient to use a description in terms of the effective Hamiltonian $H_{F}$. However, it is notoriously difficult to calculate $H_{F}$ or the exact time-evolution operator $U(t)$ for interacting systems, so generally one uses expansion techniques to find an approximate, effective Hamiltonian in the high-frequency limit. These techniques include the Magnus expansion [71-73], rotating frames [53], and many more [20,67,74-80]. Unfortunately, these methods do not produce a cleanly convergent expansion series for general systems. Instead, they are asymptotic expansions, subject to an optimal cutoff order that prevents them (in principle) from reaching into the lower-frequency regimes $[53,71]$. By this statement, we do not mean to imply that methods such as ours may not be subject to their own cutoffs but that these cutoffs may differ [81]. Whether this is the case for the exact version of the flow equations in this paper is a matter that still has to be determined.

One of the more controlled descriptions of a system occurs in the quasiequilibrium regime, $W \ll \hbar \omega \ll \Delta$, where $W$ is the bandwidth of the system, $\omega$ the driving frequency ( $\hbar$ is Planck's constant), and $\Delta$ is the gap to the continuum of higher energy states. While this separation of energy scales is quite feasible in cold atom systems, it is harder to reach in solid-state systems. Mott insulators are the most promising class of systems in this regard, but even in this case, the range of frequencies is limited since we typically have $W \sim 1 \mathrm{eV}$ and $\Delta \sim 1 \mathrm{eV}$, which are of the same order of magnitude. In addition, lower-frequency regimes are required for certain topological phases [18] and are of interest in cold atom systems [82,83] and in the study of thermalization [84]. Hence, techniques to handle lower frequencies are needed.

In this paper, we improve on the limitations of previous methods and provide better access to lower frequencies and higher driving strengths. To achieve this goal, we introduce a formalism to remove the time-dependent part of a Hamiltonian using infinitesimal unitary transformations. This results in flow equations for different couplings, reminiscent of renormalization group calculations [85] and Wegner's flow equation approach to diagonalizing Hamiltonians [86,87]. There has also recently been progress in using the Wegner flow to describe the time evolution of a many-body localized system [88], which however still requires the solution of flow equations for each time step-a problem we avoid in our construction.

We note that while a flow equation method for finding effective Floquet Hamiltonians exists in the literature, it uses an approximate version of the Wegner generator (keeping only terms proportional to $\omega$ in the generator) [89] in Sambe space [90], where the approximation brings up a question as to the range of validity. Our method differs in that we do not need to introduce Sambe space, and our generator is obtained in a constructive manner and differs completely from the Wegner generator. For our method, we describe both the exact flow equations and ways to approximate them. We apply our method to the SchwingerRabi model of a single spin in a magnetic field and also to four different spin chain Hamiltonians: (i) an integrable $X Y$ model with antisymmetric exchange, (ii, iii) two integrability-breaking extensions of a $J_{1}-J_{2}$-type $X X Z$ model [91], and (iv) the transverse field Ising model.

The extended $X Y$ model is driven by a transverse magnetic field, the first $J_{1}-J_{2}$-type $X X Z$ model is driven locally by a magnetic field in the $x$ direction, and the second is driven by a nearest-neighbor Ising exchange interaction, resulting in a time-dependent $J_{1}-J_{2}$ model $[92,93]$. For the transverse field Ising model, we consider (i) a harmonic driving case and (ii) a case where the time-evolution operator factorizes into two matrix exponentials, which allows us to find a family of different resummations of the Baker-Campbell-Hausdorff (BCH) identity. This observation leaves open the question of how to construct the optimal effective Hamiltonian for a given time-evolution operator (the reverse of the usual situation in which one seeks the optimal time-evolution-operator approximation for a given Hamiltonian).

In this paper, we study the time evolution of the exact models and the effective models obtained in our approach. We compare our results with those obtained by the Magnus expansion. The integrability-breaking models are studied numerically using full exact diagonalization, which provides an unbiased test of the validity of our approach. We find that our flow method generally outperforms the Magnus expansion, with significantly greater accuracy as the resonant regime is approached, as well as in the case when the time-dependent term in the Hamiltonian is large. Both of these cases are of direct physical relevance and interest. Our method thus opens new possibilities in the analytical and numerical simulation of time-dependent quantum many-particle systems and will facilitate the search for novel prethermal and nonequilibrium regimes.

Our paper is organized as follows. In Sec. II, we develop the general flow equation formalism and discuss its 
structure and approximations. In Sec. III, we relate the general results obtained from the flow equation approach to various high-frequency expansions used in the literature. In Sec. IV, we test the flow equations on an exactly solvable two-level system and discuss in detail the properties of the fixed points of the flow equations and their stability. This discussion is continued in Sec. V for a many-body system studied via a truncated ansatz where we show it outperforms a high-frequency Magnus expansion and rotating wave approximation. In Sec. VI, we introduce four different one-dimensional spin chain Hamiltonians that we use to assess the performance of the approximate method described in Sec. III. In Sec. VII, we summarize our results for the different models. In Sec. VIII, we compare our results to a resummation of the Baker-Campbell-Hausdorff identity that was of recent interest [81]. We also show what advantages our approach has over a standard rotating frame approximation-namely, that it can be truncated when a rotating frame transformation is not practically possible and that it still performs well under these circumstances. In Sec. IX, we present our main conclusions. Various technical details and formulas appear in the Appendixes.

\section{GENERAL FORMALISM}

We take the Schrödinger equation of a periodically driven many-particle system as our starting point. Following Ref. [53], the Hamiltonian $H(t)$ is split into a constant part $H_{0}=(1 / T) \int_{0}^{T} d t H(t)$ and a time-periodic term $V(t)=(1 / T) \int_{0}^{T} d t_{1}\left(H(t)-H\left(t_{1}\right)\right)$ that averages to zero over one period, $(1 / T) \int_{0}^{T} d t V(t)=0$. Thus, the timedependent Schrödinger equation takes the form

$$
i \partial_{t}\left|\psi_{0}\right\rangle=\left(H_{0}+V(t)\right)\left|\psi_{0}\right\rangle
$$

where we have set Planck's reduced constant $\hbar=1$.

We introduce a unitary transformation, $U=e^{\delta \Omega(t)}$, generated by an as-yet undetermined quantity $\delta \Omega$ that will be chosen to reduce the time-dependent term $V(t)$. The $\delta$ in front of the $\Omega$ indicates that we keep the generator infinitesimal, which ensures that the exponential can be safely expanded to lowest order.

Let us now introduce a new wave function $\left|\phi_{\delta s}\right\rangle=$ $U^{\dagger}\left|\psi_{0}\right\rangle=[1-\delta \Omega(t)]\left|\psi_{0}\right\rangle$ and act with $U(t)^{\dagger}=1-\delta \Omega(t)$ (to leading order in $\delta \Omega$ ) from the left on the Schrödinger equation. This new wave function now fulfills the modified Schrödinger equation (keeping lowest order in $\delta \Omega$ only),

$$
i \partial_{t}\left|\phi_{\delta s}\right\rangle=\left(H(t)-i \partial_{t} \delta \Omega(t)-[\delta \Omega(t), H(t)]\right)\left|\phi_{\delta s}\right\rangle .
$$

One may read off a new Hamiltonian, which, since $\delta \Omega$ is infinitesimal, can be written as

$$
\tilde{H}(t)=H(t)-i \partial_{t} \delta \Omega(t)-[\delta \Omega(t), H(t)] .
$$

Up to this point, this treatment coincides with the use of time-dependent generators [94]. However, we now choose $\delta \Omega$ very different from the Wegner generator. We choose it such that it reduces the time-dependent part of the Hamiltonian $V(t) \rightarrow(1-\delta s) V(t)$ by some infinitesimal value $\delta s$,

$$
\delta \Omega=-\frac{i}{T} \delta s \int_{0}^{t} d t_{1} \int_{0}^{T} d t_{2}\left(H\left(t_{1}\right)-H\left(t_{2}\right)\right),
$$

where the generator in Eq. (4) also has the nice property that it vanishes at stroboscopic times $T$. Therefore, at stroboscopic times, expectation values $\langle\hat{O}\rangle$ of operators $\hat{O}$ can be calculated without a change of basis. The behavior at other times can be found by applying the unitary transformation to the operator $\hat{O}$.

One could now repeat the procedure of splitting the Hamiltonian into a constant and a time-average zero part and then apply this infinitesimal unitary transformation to find the Floquet Hamiltonian after an infinite amount of steps (or an approximation to it by stopping after a finite amount of steps). To simplify the process, we recognize that one can track the progress of the unitary transformations by a single flow parameter $s$. To do so, we extend the functional dependencies of the Hamiltonian to include this parameter, replacing $H(t) \rightarrow H(s, t)$ and $\tilde{H}(t) \rightarrow$ $H(s+\delta s, t)$. Note that $H(s, t)$ represents a family of effective Hamiltonians interpolating between a starting Hamiltonian $H(0, t)$ and a Hamiltonian $H(\infty, t)$. Here, $H(\infty, t)$ is the Floquet Hamiltonian $H_{F}$ if $V(\infty, t)=0$. It seems plausible that $V(\infty, t)=0$; we find this to be true in an explicit example and some limiting cases, but it remains to be shown rigorously. We set appropriate boundary conditions by enforcing that $s=0$ corresponds to the initial, nontransformed Hamiltonian.

With this notation, Eq. (3) takes the form

$$
\begin{aligned}
H(s+\delta s, t)= & H(s, t)-\delta s V(s, t) \\
& +i \delta s \int_{0}^{t} d t_{1}\left[V\left(s, t_{1}\right), H(s, t)\right],
\end{aligned}
$$

with $V(s, t)=(1 / T) \int_{0}^{T} d t_{1}\left(H(s, t)-H\left(s, t_{1}\right)\right)$. One may note that this leaves a residual time dependence of $\delta s[V(t), H(t)]$ in Eq. (3), which is small in magnitude if $\delta s$ is small.

Taylor expanding the left-hand side since $\delta s$ is infinitesimal, we find

$$
\frac{d H(s, t)}{d s}=-V(s, t)+i \int_{0}^{t} d t_{1}\left[V\left(s, t_{1}\right), H(s, t)\right]
$$

which is a central result of this work. We refer to Eq. (6) as the exact flow equation. This equation is similar in spirit to the infinitesimal unitary transforms that Wegner [86] employs to diagonalize an interacting Hamiltonian in the equilibrium case.

One can readily see that Eq. (6) has a fixed point with the desired property $V(s, t)=0$. This fixed point is guaranteed 
to be stable for sufficiently large $\omega$ because in this case the commutator term can be neglected. Under these circumstances, the time-independent parts of $H(s, t)$ remain unchanged. More precisely, Eq. (6) then reduces to

$$
\frac{d V(s, t)}{d s} \approx-V(s, t)
$$

which trivially has the stable fixed point $V(s, t)=0$ since $V(s, t)$ for this case can be treated like a scalar.

But what about smaller $\omega$ ? Because an analytic understanding is difficult to achieve, we discuss this in the context of an explicit example in Sec. IV. While our discussion gives a mechanism by which the fixed points can be stable, in general, it does not give a rigorous proof.

How should one interpret the flow of $s$ in Eq. (6)? Note that $H(s, t)$ is a Hamiltonian and therefore a linear sum of the various energy contributions, and it can be expressed as a sum of linear operators with coefficients $c_{i}(s, t)$, $H(s, t)=\sum_{i} c_{i}(s, t) \hat{O}_{i}$ (similar in spirit to a LandauGinzburg energy functional). The $\hat{O}_{i}$ operators are nothing other than kinetic and potential energy terms appearing in a Hamiltonian, such as a hopping term $c_{i}^{\dagger} c_{j}$ in a lattice model, an interaction term $n_{i \uparrow} n_{i \downarrow}$ on a lattice, or a multiplespin term $\left(\vec{S}_{i} \cdot \vec{S}_{j}\right)\left(\vec{S}_{k} \cdot \vec{S}_{k}\right)$ in a spin model, among many other possibilities. The coefficients $c_{i}(s, t)$ describe the coupling constants (strength) of these terms.

This mathematical structure of $H(s, t)=\sum_{i} c_{i}(s, t) \hat{O}_{i}$ in turn also implies that $-V(s, t)+i \int_{0}^{t} d t_{1}\left[V\left(s, t_{1}\right), H(s, t)\right]=$ $-\sum_{i} g_{i}\left(t,\left[c_{j}\left(s, t^{\prime}\right)\right]\right) \hat{O}_{i}$. Here, $g_{i}$ has a functional dependence on the $c_{j}\left(s, t^{\prime}\right)$ with $t^{\prime} \in[0, T]$ because $V(s, t)$ itself depends on the $c_{j}(s, t)$ and it appears under an integral.

One may therefore write Eq. (6) as

$$
\frac{d c_{i}(s, t)}{d s}=-g_{i}\left(t,\left[c_{j}\left(s, t^{\prime}\right)\right]\right) ; \quad t^{\prime} \in[0, T],
$$

which is just a flow equation for the coupling parameters $c_{i}(s, t)$ at different times. Note that the set of operators $\hat{O}_{i}$ may include both the original operators and ones generated from the kinetic and potential energy terms of the original Hamiltonian, Eq. (1), as the Hamiltonian flows. In general, new terms are generated such as hopping and interaction terms that involve more and more sites of a lattice as the order of the transformation increases. These new terms can, in principle, change the balance of kinetic and potential energy in the effective time-independent Hamiltonian and therefore may lead to new physical regimes for a periodically driven many-particle quantum system. The reason we write the flow equations in this form is to emphasize that Eq. (6) actually describes couplings that flow as we reduce the time dependence and to show how this operator equation corresponds to a numerically tractable scheme to determine couplings.

\section{APPROXIMATIONS TO THE FLOW EQUATIONS}

It is important to note that Eq. (6) offers a convenient starting point to approximate the Floquet Hamiltonian. In particular, it allows us to improve on the various highfrequency expansions of the Floquet Hamiltonian that have appeared in the literature. As an example, we can find an analytically tractable equation if we set $s=0$ only for the terms $V(s, t)$. This corresponds to removing the original time-dependent part $V(t)$ from the Hamiltonian via the rotating frame transformation [95] $e^{-i \int_{0}^{t} d t V(t)}$ while generating other new time dependences. [This approximation is made for convenience. Indeed, in the following section, we present an example in which we exactly solve Eq. (6) without taking $s=0$ in $V(s, t)]$. To ensure that this approximation actually corresponds to the aforementioned unitary transformation, we also need to restrict the range of $s$ to $[0,1]$ rather than the previous $[0, \infty)$.

Let us justify this approximation slightly more carefully by using an analogy. One may notice that Eq. (6) is very similar in structure to the classical problem of a first-order differential equation,

$$
\frac{d f(t)}{d t}=g(t, f(t))
$$

where $g(t, f(t))$ would correspond to $-V(s, t)+$ $i \int_{0}^{t} d t_{1}\left[V\left(s, t_{1}\right), H(s, t)\right]$ in our case and all the couplings in $H$ and $V$ correspond to $f(t)$.

A standard method of solving this class of problems [96] is plugging in the initial condition $f(t) \rightarrow f_{0}=f(t=0)$ on the right-hand side. Integrating both sides of the equation, one finds a first approximation to $f(t)$, which we call $f_{1}(t)$. One may then repeat the procedure and plug successive approximations $f_{n}(t)$ into the right-hand side. This procedure is called Picard iteration. In our case, it is the same as replacing $V(s, t) \rightarrow V(0, t)$ and $H(s, t) \rightarrow H(0, t)$.

A variant of Picard iteration that quite often works better is to only set $f(t)=f_{n}(t)$ in some places of $g(t, f(t))$ but keep it as $f(t)$ in others. This is a particularly helpful improvement when it is done in such a way that some symmetries are explicitly kept that would otherwise be destroyed [71]. For our case, if we only replace the first two $V(s, t) \rightarrow V(0, t)$ but keep $H(s, t)$, then we find approximate flow equations,

$\frac{d H(s, t)}{d s}=-V(0, t)+i \int_{0}^{t} d t_{1}\left[V\left(0, t_{1}\right), H(s, t)\right]$

where $s$ is set to run from 0 to 1 . As required above, this still implements a unitary transformation, which can be seen explicitly by reconstructing Eq. (10) from a unitary transformation. 
Introducing the dependence on the flow parameter $s$, Eq. (3) reads

$$
H(s+\delta s, t)=H(s, t)-i \partial_{t} \delta \Omega(s, t)-[\delta \Omega(s, t), H(s, t)] .
$$

One may plug in the manifestly anti-hermitian generator $\delta \Omega(s, t) \equiv \delta \Omega(0, t)=-i \delta s \int_{0}^{t} d t V(0, t)$, corresponding to a unitary transformation $U$. The result is Eq. (10). Therefore, making such an approximation is a particularly convenient improvement on a Picard iteration.

One may ask why $s$ should run from 0 to 1 as claimed above. One reason for this is that, in the lowest-order improved Picard iteration, we neglect terms that are proportional to $s$. Neglecting such terms is only justified if $s \leq 1$. Therefore, we let the flow parameter run from zero to 1 . If we reach a fixed point in this range of values or come close to it, then it is a good approximation. Letting $s$ run to higher values would not be justified and may yield a bad result. Another reason we apply this approach is because we know that for infinite frequencies, one reaches a stable fixed point for $s=1$. This result can be seen easily because Eq. (10) is then approximately given as

$$
\frac{d V(s, t)}{d s} \approx-V(0, t)
$$

This procedure also works well in other cases because, often at $s=1$, one may be close to an unstable fixed point (see, e.g., Fig. 1). We should also mention that the multitude of different possible fixed points [all $V(s, t)=0$ ] and their corresponding $s$ value makes it difficult to estimate the size of the error from letting $s$ only run from 0 to 1 . After all, $s=1$ is often close to a fixed point, but there may be more fixed points further out (for larger values of $s$ ). We see this

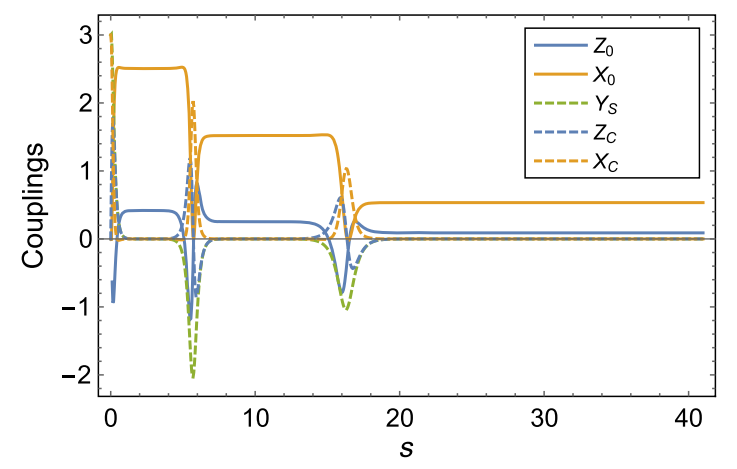

FIG. 1. The couplings as a function of flow parameter $s$ for the Hamiltonian in Eq. (19) with $B_{p}=3, B_{z}=1$, and $\omega=1$ in Eq. (17). This case corresponds to a low-frequency regime. Note that while the couplings exhibit a nontrivial dependence on $s$ until sufficiently large $s$, the unitary evolution remains stable down to small frequencies, as seen in the red curve (exact flow) in Fig. 3. The couplings after the range of the plot do not change within the limits of the line thicknesses. explicitly in the next section where we work with the exact flow equations.

Now, let us return to discussing Eq. (10). One finds that this equation can also be rewritten in terms of coupling constants as

$$
\frac{d c_{i}(s, t)}{d s}=-g_{i}\left(t, c_{j}(s, t)\right)
$$

where one can write $g_{i}\left(t, c_{j}(s, t)\right)=\sum_{j} \gamma_{i j}(t) c_{j}(s, t)$ as a linear combination of couplings $c_{j}(s, t)$. We are therefore left with a first-order linear differential equation that does not couple coefficients $c_{j}$ at different times. The more complicated structure of a functional in the $c_{i}$ is no longer present. The effective time-independent Hamiltonian is then given by

$$
H_{\mathrm{eff}}=\sum_{i} \bar{c}_{i} \hat{O}_{i}
$$

with $\bar{c}_{i}=(1 / T) \int_{0}^{T} d t c_{i}(1, t)$, where we have taken an average over one period, which is physically meaningful if one is only looking at stroboscopic times. If one is interested in micromotions, one could, in principle, retain the time dependence of $c_{i}(1, t)$-the important "flow" having been taken into account in the parameter $s$, which has now been set to unity.

The approximation made in Eq. (10), setting $s=0$ in $V(s, t)$, does not make any implicit assumptions, such as $V(t)$ is small. By contrast, many other high-frequency approximations do make the assumption of smallness. As a result, our approach, like the rotating frame approximation, works especially well in the limit of strong $V(t)$. We demonstrate this explicitly in later sections of this work.

It is important to pause for a moment and stress the advantages our approximate method, Eq. (10), offers over a rotating frame approximation, if the latter is carried out exactly. First, if the driving is complicated, it is often not possible to calculate the matrix exponential needed for a rotating frame approximation or the rotation induced on operators by such a matrix exponential because it will generate infinitely many components of the operator algebra. This is indeed the case with one of our example models, namely, the square-wave driven Ising model that we discuss later. In this case, our method allows one to keep all orders in $1 / \omega$ with a truncated ansatz for the Hamiltonian. This method performs well, as can be seen in the plot in Sec. VIII (Fig. 14).

It is also important to recognize that, even if a rotating frame approximation can be done exactly, usually most terms in the Hamiltonian become time dependent. In most cases, this makes a second rotating frame approximation impossible. Our method allows one to avoid this issue by truncating the ansatz Hamiltonian. Lastly, in some cases, one would like to prevent the generation of any new terms 
and see what happens to the coupling constants of a restricted set of terms. Thus, our method provides a convenient starting point for many different approximation schemes.

We would also like to stress that Eq. (10) implements a unitary transformation exactly. Its solution therefore still retains the full information of the original Hamiltonian. In this paper, we are content with discussing results from the first-order iteration only. Again, the formalism we present here lays the groundwork for further development of approximation schemes.

Let us explicitly relate the first-order iteration to the more common high-frequency approximations. For the moment, neglect $\int_{0}^{t} d t_{1}\left[V\left(0, t_{1}\right), H(s, t)\right]$, which assumes that all couplings in the Hamiltonian are negligible compared to the driving frequency. This approximation is common to many of the high-frequency approximations. We then find that

$$
H(s, t) \approx H(0, t)-s V(t) .
$$

Inserting this back into Eq. (10) and taking a time average, we find

$H(1, t) \approx H_{0}+\frac{i}{T} \int_{0}^{T} d t \int_{0}^{t} d t_{1}\left[V\left(t_{1}\right), H_{0}+\frac{1}{2} V(t)\right]$,

which is the lowest order of many common high-frequency approximations. Hence, our approximation agrees with other approximations in the high-frequency limit.

One should also note that there are other ways to approximately solve the exact flow equation by directly working with Eq. (6) and a truncated ansatz rather than solving Eq. (10). We show this in one example in Sec. V and find that it indeed offers an improvement to the methods above (rotating frame and high-frequency expansion) and opens the door to many semianalytical schemes.

Next, we turn to an application of our method to a number of different Hamiltonians and compare our results with other approaches. We find that the method nearly always provides more accurate evolution than other approximations, and in many cases, our method works substantially better, particularly as the strong coupling resonant regime is approached. This is also true if we solve Eq. (10) with a truncated ansatz like one of the cases in Sec. VIII.

\section{FIXED-POINT STABILITY AND PROPERTIES OF EXACT FLOW EQUATIONS}

Because it is difficult to discuss the stability of the flow equations in Eq. (10) analytically in full generality, we consider a simple example model where the exact flow equations can be written down explicitly. This model will allow us to identify a mechanism that makes the fixed point stable. It is conjectured-but, we stress, not rigorously proven - that this mechanism will persist even for more complicated systems. In Sec. V, this conjecture will be further supported. The current section serves as a means to gain some insight into how the flow equations work. We consider the Schwinger-Rabi model of a spin in a rotating magnetic field,

$$
H=B_{z} \sigma_{z}+B_{p}\left(\sin (\omega t) \sigma_{y}+\cos (\omega t) \sigma_{x}\right) .
$$

For this model, the Floquet Hamiltonian

$$
H_{\mathrm{F}}=-\frac{\omega}{2}+B_{p} \sigma_{x}+\left(B_{z}-\frac{\omega}{2}\right) \sigma_{z}
$$

can be found for all frequencies (see, for instance, Ref. [97]).

Let us discuss how the flow equations apply to this model. After repeatedly inserting the form of the original Hamiltonian in our exact flow equations in Eq. (6) (always including newly generated terms), we find that the Hamiltonian $H(s, t)$ takes the form

$$
\begin{aligned}
H(s, t)= & Z_{0}(s) \sigma_{z}+X_{0}(s) \sigma_{x}+Y_{S}(s) \sin (\omega t) \sigma_{y} \\
& +X_{C}(s) \cos (\omega t) \sigma_{x}+Z_{C}(s) \cos (\omega t) \sigma_{z}
\end{aligned}
$$

and the flow equations for the couplings $\left\{Z_{0}, X_{0}, Y_{S}\right.$, $\left.X_{C}, Z_{C}\right\}$ are given as

$$
\begin{aligned}
Z_{0}^{\prime}(s) & =\frac{2 Y_{S}(s)\left(X_{0}(s)-X_{C}(s)\right)}{\omega}, \\
X_{0}^{\prime}(s) & =\frac{2 Y_{S}(s)\left(Z_{C}(s)-Z_{0}(s)\right)}{\omega}, \\
Y_{S}^{\prime}(s) & =\frac{2\left(Z_{0}(s) X_{C}(s)-Z_{C}(s) X_{0}(s)\right)}{\omega}-Y_{S}(s), \\
Z_{C}^{\prime}(s) & =\frac{2 Y_{S}(s)\left(X_{C}(s)-X_{0}(s)\right)}{\omega}-Z_{C}(s), \\
X_{C}^{\prime}(s) & =\frac{2 Y_{S}(s)\left(Z_{0}(s)-Z_{C}(s)\right)}{\omega}-X_{C}(s)
\end{aligned}
$$

(where the ' denotes the derivative with respect to $s$ ) with initial conditions

$$
\begin{aligned}
& Z_{0}(0)=B_{z}, \quad Y_{S}(0)=X_{C}(0)=B_{p}, \\
& Z_{C}(0)=X_{0}(0)=0 .
\end{aligned}
$$

As expected from Eq. (6), we find that the fixed point is $Y_{S}=X_{C}=Z_{C}=0$, with arbitrary $Z_{0}$ and $X_{0}$. This is the only fixed point. For this fixed point, we may carry out a stability analysis. In other words, we expand Eq. (20) around the fixed point to find linearized equations $\mathbf{C}^{\prime}(s)=J \mathbf{C}(s)$, where $\mathbf{C}=\left\{Z_{0}, X_{0}, Y_{S}, X_{C}, Z_{C}\right\}$ is a vector of the couplings. The eigenvalues of the corresponding Jacobian $J$ are given as 
$\lambda_{1}=\lambda_{2}=0 ; \quad \lambda_{3,4}=-1 \pm \frac{2}{\omega} \sqrt{Z_{0}^{2}+X_{0}^{2}} ; \quad \lambda_{5}=-1$.

It would appear that not all eigenvalues are guaranteed to be nonpositive. In particular, one of the eigenvalues $\lambda_{3,4}$ could be positive, which would imply that the fixed point is unstable and that the flow equations break down. If the form of the Hamiltonian at the fixed point reproduces that of Eq. (18), this could indeed be the case since $Z_{0}$ and $X_{0}$ would be finite for arbitrarily small $\omega$. One might thus expect that flow equations would be unable to reach a stable fixed point for low enough frequencies. However, this outcome is avoided.

To see how this works, recall that the Floquet Hamiltonian $H_{F}$ is determined only up to some phase by

$$
e^{-i H_{F} \frac{2 \pi}{\omega}}=U\left(\frac{2 \pi}{\omega}\right)
$$

where $U(t)$ is the time-evolution operator. This means that there are many different expressions for $H_{F}$ that would be valid branches of the matrix logarithm of both sides of Eq. (23). For very small $\omega$, a valid $H_{F}$ could be chosen to be very small. Let us see what happens explicitly for our flow equations. Namely, let us choose couplings such that Eq. (18) would correspond to an unstable fixed point. How these couplings evolve under the flow equations can be seen in Fig. 1.

In Fig. 1 we see that the couplings $Z_{0}$ and $X_{0}$ closely approach a fixed point $V(s, t)=0$ several times but it is not stable at first. However they keep shrinking until a stable fixed point is reached. The matrix logarithm $\log (U(T))$ has branches with relatively small $H_{F}$, and the couplings continue flowing until a branch with sufficiently small couplings to have a stable fixed point is reached. In the language of the exact flow equations, Eq. (6), there exists a branch of the matrix logarithm $\log (U(T))$ such that $H(s, t)$ becomes sufficiently small that the commutator $\int_{0}^{t} d t_{1}\left[V\left(s, t_{1}\right), H(s, t)\right]$ becomes negligible compared to $V(s, t)$, and therefore a stable fixed point is reached. We are able to observe this effect in all cases that we studied, and it is plausible that this could be a general mechanism that leads to stable fixed points in our flow equations. This is illustrated in Fig. 2.

From Figs. 1 and 2, one may suspect numerical issues. However, this is not the case. Rather, the oscillations stem from the fact that the flow equations do not consistently stay on one branch of the matrix logarithm for $H_{F}$. Flowing to a stable fixed point means choosing the branch of the matrix logarithm that corresponds to a stable fixed point.

Indeed, if we take the time-independent couplings in Fig. 2 to calculate the time-evolution operator at stroboscopic times and compare it to the time-evolution operator calculated via the standard method of a Trotter expansion,

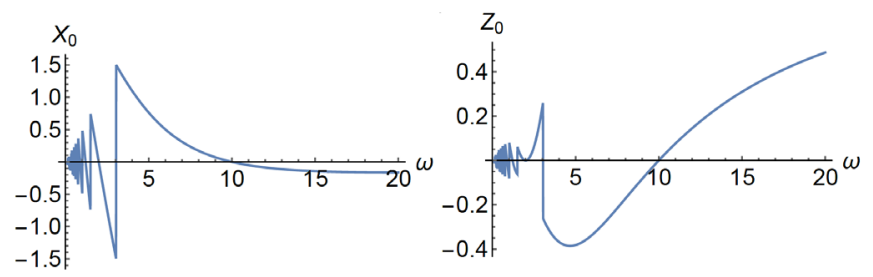

FIG. 2. The nonzero couplings as a function of frequency $\omega$ at the end of the flow (large $s$ values in Fig. 1) for $B_{p}=3, B_{z}=1$. Note that in spite of the rapid oscillations for small $\omega$, the resultant unitary evolution remains stable, as seen in the red curve (exact flow) in Fig. 3.

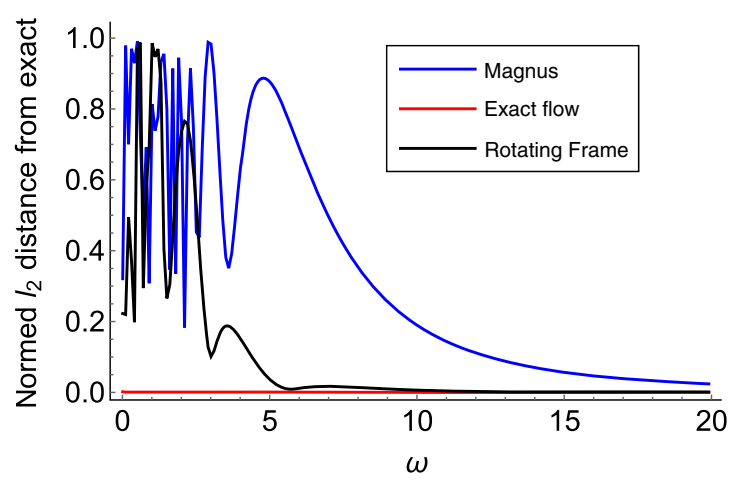

FIG. 3. Plot of the $l_{2}$ distance between the time-evolution operator found by a Trotter expansion and the exact timeevolution operator obtained by exactly solving the flow equations in Eq. (6) given by Eq. (20) (red line), the Magnus expansion (blue line), and the rotating frame approximation given by Eq. (10) (black line).

we find them to be identical. More specifically, we calculate the $l_{2}$ distance between two unitary operators,

$\frac{1}{2 \sqrt{D_{\text {dim }}}}\left\|U_{1}(T)-U_{2}(T)\right\|_{\text {Frob }} ; \quad\|A\|_{\text {Frob }}=\sqrt{\operatorname{tr} A A^{\dagger}}$,

which was normed such that it takes values between 0 and 1 ( $D_{\text {dim }}$ is the dimension of the Hilbert space), where 1 corresponds to the maximum distance between two unitary operators and 0 to agreement between the two operators. A comparison is shown in Fig. 3. Details of the rotating frame approximation and Magnus expansion are given in Appendix A. We find that the exact flow equationsdespite the couplings rapidly changing-fully agree with the Trotter expansion, as they should. The wildly jumping couplings are therefore not a numerical artifact.

\section{EXACT FLOW EQUATIONS WITH A TRUNCATED ANSATZ}

In this section, we discuss how the results from the previous section seem to be quite generic by considering a 
many-body system. We limit ourselves to a specific, strongly driven Ising model given by

$$
H(t)=\sum_{i}\left[\sigma_{i}^{z} \sigma_{i+1}^{z}+4 \cos (\omega t) \sigma_{i}^{z}+4 \sin (\omega t) \sigma_{i}^{x}\right] .
$$

We choose this Hamiltonian because (i) it has a relatively strong external drive, (ii) it has a time-dependent term that does not commute with itself at different times, and (iii) the time dependence is convenient for studying the flow equations. One may find flow equations by making the truncated ansatz,

$$
H(s)=H_{0}(s)+e^{i \omega t} H_{+}(s)+e^{-i \omega t} H_{-}(s),
$$

with

$$
\begin{aligned}
H_{a}= & \sum_{i}\left[C_{x}^{a} \sigma_{i}^{x}+C_{y}^{a} \sigma_{i}^{y}+C_{z}^{a} \sigma_{i}^{z}+C_{x x}^{a} \sigma_{i}^{x} \sigma_{i+1}^{x}\right. \\
& +C_{x y}^{a}\left(\sigma_{i}^{x} \sigma_{i+1}^{y}+\sigma_{i}^{x} \sigma_{i-1}^{y}\right)+C_{y y}^{a} \sigma_{i}^{y} \sigma_{i+1}^{y} \\
& +C_{x z}^{a}\left(\sigma_{i}^{x} \sigma_{i+1}^{z}+\sigma_{i}^{x} \sigma_{i-1}^{z}\right)+C_{z z}^{a} \sigma_{i}^{z} \sigma_{i+1}^{z} \\
& \left.+C_{y z}^{a}\left(\sigma_{i}^{y} \sigma_{i+1}^{z}+\sigma_{i}^{y} \sigma_{i-1}^{z}\right)+C_{x z z}^{a} \sigma_{i}^{x} \sigma_{i-1}^{z} \sigma_{i+1}^{z}\right],
\end{aligned}
$$

where $a \in\{0,+,-\}$ and the $s$ dependence of the coupling constants $C^{a}$ was dropped for notational simplicity. We do not discuss the specific form of the flow equations here because they are rather complicated and not insightful. Instead, we first look at how some of the couplings behave for this system. Specifically, let us look at one representative coupling as a function of flow parameter $s$. As one may see from Fig. 4, the coupling constant $C_{z}^{+}$behaves similarly to the ones in Fig. 2 for the two-level system we solved exactly in the previous section. In particular, the coupling constant nearly approaches zero for the fixed point multiple times before, eventually, a stable fixed point is reached. This result strengthens our interpretation that our method might be kept stable by the mechanism we provided in Sec. IV.

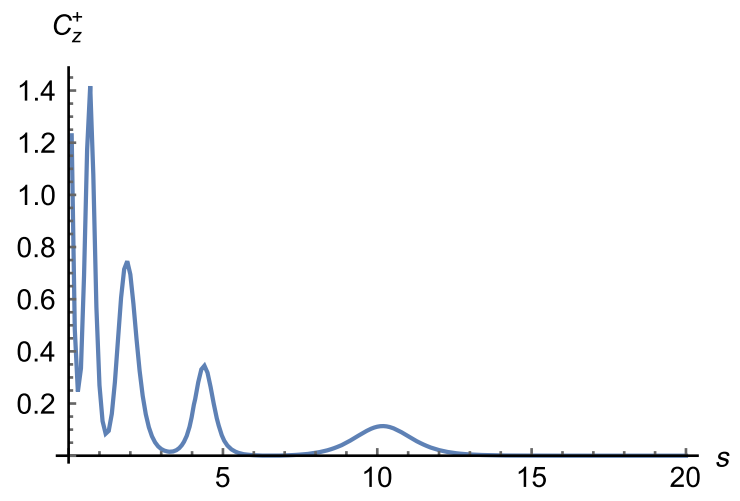

FIG. 4. Coupling constant $C_{z}^{+}$as a function of flow parameter $s$ plotted for $\omega=1.2$.

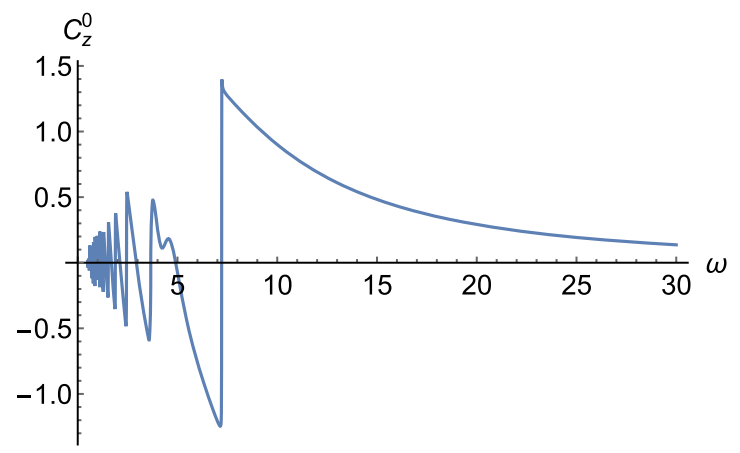

FIG. 5. Plot of the coupling constant $C_{z}^{0}$ as a function of $\omega$ for the flow equations solved up to a point $s=2000000$, which is long after the fixed point has been reached.

To get further evidence for this interpretation, we plot in Fig. 5 one of the couplings as a function of $\omega$ and again find it to be consistent with the mechanism we proposed in Sec. IV and illustrated in Fig. 2. We stress that this is not a rigorous proof of our understanding of how the flow equations manage to converge, but it is does provide good evidence for the general structure of the convergence.

Let us now discuss these results further. One finds numerically that by letting $s \rightarrow \infty$, only certain terms survive. Namely, as expected from the fixed point $C_{i}^{ \pm} \rightarrow 0$, one is left with

$$
\begin{aligned}
H(s \rightarrow \infty)= & \sum_{i}\left[C_{y} \sigma_{i}^{y}+C_{z} \sigma_{i}^{z}+C_{x x} \sigma_{i}^{x} \sigma_{i+1}^{x}+C_{y y} \sigma_{i}^{y} \sigma_{i+1}^{y}\right. \\
& \left.+C_{z z} \sigma_{i}^{z} \sigma_{i+1}^{z}+C_{y z}\left(\sigma_{i}^{y} \sigma_{i+1}^{z}+\sigma_{i}^{y} \sigma_{i-1}^{z}\right)\right] .
\end{aligned}
$$

The couplings in the range $\omega \in[8,40]$ are well approximated by

$$
\begin{aligned}
& C_{x x}^{0}=\frac{0.99}{\omega}-0.12+0.006 \omega-0.00013 \omega^{2}, \\
& C_{y y}^{0}=\frac{4.87}{\omega}+0.18-0.021 \omega+0.0006 \omega^{2}, \\
& C_{z z}^{0}=-\frac{5.75}{\omega}+0.87+0.022 \omega-0.00067 \omega^{2}, \\
& C_{y z}=-\frac{52.07}{\omega^{2}}+\frac{7.35}{\omega}+0.1-0.0017 \omega, \\
& C_{y}^{0}=\frac{78.2}{\omega^{2}}-\frac{18.87}{\omega}+0.03+0.00013 \omega, \\
& C_{z}^{0}=\frac{13.24}{\omega}-0.49+0.0066 \omega-0.000011 \omega^{2} .
\end{aligned}
$$

Such fitted couplings allow for a semianalytic understanding in some cases. One should note that for smaller $\omega$, the expressions become much more complicated because of the nonanalytic behavior of the couplings as seen in Fig. 5.

Let us show below how well our approximation [also using results for smaller $\omega$ and not just the expression in Eq. (29)] performs when compared to the rotating frame 


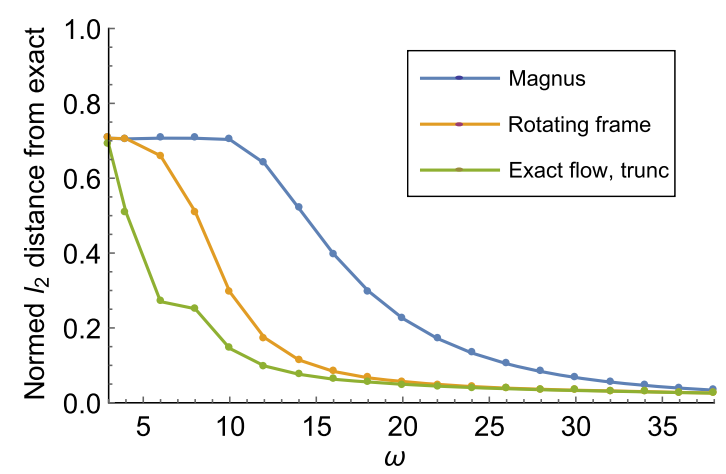

FIG. 6. Plot of the $l_{2}$ distance between the exact time-evolution operator and the Magnus expansion (blue line), rotating frame approximation (orange line), and the solution of the exact flow equations but with a truncated ansatz (green line). The system size is $L=14$ sites.

approximation and the Magnus expansion. We do not explicitly give the expressions for the couplings in the Magnus expansion and the rotating frame approximation because they are cumbersome and do not provide much physical insight. Instead, we refer the interested reader to Ref. [98]. From Fig. 6, one finds that the flow equations (with a truncated ansatz) perform better than both the Magnus expansion and the rotating frame approximation. To stress that the comparison to the rotating frame approximation is a fair one, we note that the operators in Eq. (28) are the same as those appearing within the rotating frame approximation. From this example, one sees that the exact flow equations allow one to find better coefficients than those afforded by the rotating frame approximation.

\section{EXAMPLE MODELS}

To demonstrate the power and validity of the flow equation approach for a wider range of many-body systems, we next consider a selection of quantum spin chain $\left(S=\frac{1}{2}\right)$ models. Recall that the spin operators $S_{n}^{x, y, z}$ fulfill the commutation relations,

$$
\left[S_{m}^{j}, S_{n}^{k}\right]=i \epsilon_{j k l} \delta_{m n} S_{m}^{l}
$$

$(j, k, l \in\{x, y, z\}$ and $m, n$ label lattice sites), with the special condition for $S=\frac{1}{2}$ that

$$
\left(S_{n}^{j}\right)^{2}=\frac{1}{4} \mathbb{1}_{\mathcal{H}},
$$

where $\mathbb{1}_{\mathcal{H}}$ is the unit operator in the many-body Hilbert space. Here, $\epsilon_{j k l}$ is the fully antisymmetric tensor, and $\delta_{m n}$ is the Kronecker delta function.

In this section, we introduce four different spin models that exhibit different functional dependences of the timedependent term. The first model ( $X Y$ spin chain) is integrable and, in particular, one-particle reducible. The next two models are integrability-breaking modifications of the $X X Z$ spin chain, and the final model is a transverse field Ising model, which will be discussed independently in Sec. VIII. These models possess a range of different symmetries and forms of the driving term. They will illustrate the generality and mathematical structure of the flow equation approach.

\section{A. $X Y$ spin chain with antisymmetric exchange in a driven magnetic field}

As a first example model, we choose an $X Y$ spin chain with an antisymmetric Dzyaloshinskii-Moriya exchange interaction and a time-periodic magnetic field that both point along the $z$ axis,

$$
H(t)=H_{0}+V(t)
$$

where

$H_{0}=\sum_{i}\left(J_{x} S_{i}^{x} S_{i+1}^{x}+J_{y} S_{i}^{y} S_{i+1}^{y}+D\left(\vec{S}_{i} \times \vec{S}_{i+1}\right)_{z}+h_{0} S_{i}^{z}\right)$,

and

$$
V(t)=h \sin (\omega t) \sum_{i} S_{i}^{z}
$$

Here, $J_{x / y}$ is the strength of the exchange interaction in the $x / y$ direction, $D$ the strength of the antisymmetric exchange, $h_{0}$ the static magnetic-field strength, and $h$ the strength of the magnetic-field driving. This model has the advantage that its instantaneous Hamiltonian can be diagonalized by applying a Jordan-Wigner transformation, followed by a Bogoliubov transformation [91]. Furthermore, it has multiple coefficients, which can be varied to check the validity of our approximation based on the flow equations in a variety of cases. Note that the driving term generally does not commute with the static part of the Hamiltonian.

\section{B. $J_{1}-J_{2}$ model with a driven magnetic field in the isotropic plane}

In order to find out if a new approximation scheme is valuable for more realistic interacting systems, it is important to go beyond noninteracting models. To this end, we study the $J_{1}-J_{2}$ model $[92,93]$,

$$
H(t)=H_{0}+V(t)
$$

where 


$$
H_{0}=\sum_{n=1}^{2} \sum_{i}\left(J_{n} \mathbf{S}_{i}^{\perp} \cdot \mathbf{S}_{i+n}^{\perp}+J_{n}^{z} S_{i}^{z} S_{i+n}^{z}\right)
$$

and

$$
V(t)=\sum_{i} h(t) S_{i}^{x}
$$

with a time periodic magnetic field in the $x$ direction,

$$
h(t)=B\left\{\begin{array}{ll}
1, & 2 n \pi<\omega t<2 n \pi+\pi \\
-1, & 2 n \pi+\pi<\omega t<2(n+1) \pi
\end{array} \quad n \in \mathbb{Z},\right.
$$

where the time dependence was chosen to simplify the numerical treatment done by exact diagonalization. None of our physical conclusions-nor our flow equation method-relies on this piecewise constant form of the time dependence. It should be noted that $J_{n}$ is the strength of the $n$th neighbor exchange interaction in the isotropic plane, and $J_{n}^{z}$ is the exchange interaction in the $z$ direction. For a more compact notation, we define $\mathbf{S}_{i}^{\perp}=\left(S_{i}^{x}, S_{i}^{y}, 0\right)$. We choose this model because the external magnetic field breaks magnetization conservation, and it therefore also allows us to see if the flow equation approach works under circumstances where the driving breaks a symmetry of the static part of the Hamiltonian.

\section{C. $J_{1}-J_{2}$ model with time-dependent exchange terms}

We also apply the flow equation approach to a model in which one of the spin-spin interaction terms is time dependent. The model we consider is another $J_{1}-J_{2}$ model given by

$$
H(t)=H_{0}+V(t)
$$

where

$$
H_{0}=\sum_{i}\left[J_{1} \mathbf{S}_{i}^{\perp} \cdot \mathbf{S}_{i+1}^{\perp}+J_{1}^{z} S_{i}^{z} S_{i+1}^{z}+J_{2} \mathbf{S}_{i} \cdot \mathbf{S}_{i+2}\right]
$$

and

$$
V(t)=J_{1,0}^{z} \operatorname{sign}\left(\frac{\pi}{\omega}-t \bmod (2 \pi / \omega)\right) \sum_{i} S_{i}^{z} S_{i+1}^{z},
$$

where mod denotes modulo. In this model, the time dependence is in an interaction term.

In Sec. VIII, we consider one further spin model (Ising model) separately because the structure of the fixed-point Hamiltonian is different than the three models introduced in this section. Together, these four spin models and the example given in Sec. IV should provide a compelling picture for the generality and power of our method.

\section{RESULTS}

In this section, we study how well our flow equation approach performs compared to common high-frequency approximations. We compare the approximate timeevolution operators obtained through various approximations to the exact time-evolution operator (obtained by exact diagonalization) at stroboscopic times.

We adhere to the following procedure: We first make use of the translational invariance of our models and calculate the exact time-evolution operator $U_{\mathrm{ex}}^{k}(T)$ and the approximate time-evolution operator $U_{\text {approx }}^{k}(T)$ at different points in $k$-space (momentum space). Then, we calculate the mismatch of the approximate time-evolution operator and the exact time-evolution operator via

$$
E=\frac{1}{2 N \sqrt{D_{\mathrm{dim}}}} \sum_{k}\left\|U_{\mathrm{ex}}^{k}(T)-U_{\mathrm{approx}}^{k}(T)\right\|_{\mathrm{Frob}},
$$

which is a quantity that takes values on the interval $[0,1]$, with 0 meaning perfect agreement and 1 meaning the largest possible disagreement. Here, $D_{\text {dim }}$ is the dimensionality of the Hilbert space for any given $k$-point, $N$ is the number of $k$-points that the sum runs over, and $\|A\|_{\text {Frob }}:=$ $\sqrt{\operatorname{tr} A A^{\dagger}}$ is the Frobenius norm.

Let us motivate this quantity: For a given point in $k$-space, this quantity is just the $l_{2}$ distance, Eq. (24), between two unitary operators at this point in $k$-space divided by the maximum $l_{2}$ distance of two unitary operators. We average this quantity over all points of $k$-space. The Frobenius norm provides us with a basis-independent measure of how accurate unitary evolution of a quantum system will be with various time-independent approximations to the full timedependent Hamiltonian. Similar formulas are used in the context of quantum information science.

\section{A. $X Y$ spin chain with antisymmetric exchange}

Both the Magnus expansion (see Appendix B) and the approximation via flow equations yield an effective Hamiltonian of the form

$$
\begin{aligned}
H_{\mathrm{eff}}= & \sum_{i}\left(J_{x}^{(a)} S_{i}^{x} S_{i+1}^{x}+J_{y}^{(a)} S_{i}^{y} S_{i+1}^{y}+D_{+}^{(a)} S_{i}^{x} S_{i+1}^{y}\right. \\
& \left.+D_{-}^{(a)} S_{i}^{y} S_{i+1}^{x}+h_{0} S_{i}^{z}\right)
\end{aligned}
$$

where $a$ labels the approximation scheme, with different coupling constants for different approximation schemes. The details of the derivation are given in Appendix B.

There are newly generated terms in Eq. (43) compared to Eq. (33). We note that a suitably chosen rotation in spin space results in the original undriven Hamiltonian with $\Delta J=J_{x}-J_{y}$ modified.

The coupling constants for the leading-order Magnus expansion are 


$$
J_{x, y}^{(M)}=J_{x, y}, \quad D_{ \pm}^{(M)}= \pm D-\frac{\left(J_{x}-J_{y}\right) h}{\omega}
$$

and the results for the flow equation approach are

$$
\begin{gathered}
J_{x}^{(F)}=\frac{J_{x}+J_{y}}{2}+\frac{J_{x}-J_{y}}{2} \cos \left(\frac{2 h}{\omega}\right) J_{0}\left(\frac{2 h}{\omega}\right), \\
J_{y}^{(F)}=\frac{J_{x}+J_{y}}{2}-\frac{J_{x}-J_{y}}{2} \cos \left(\frac{2 h}{\omega}\right) J_{0}\left(\frac{2 h}{\omega}\right), \\
D_{ \pm}^{(F)}= \pm D-\frac{J_{x}-J_{y}}{2} \sin \left(\frac{2 h}{\omega}\right) J_{0}\left(\frac{2 h}{\omega}\right) .
\end{gathered}
$$

It should be emphasized that both approximations agree in the limit of $\omega \rightarrow \infty-\mathrm{a}$ general result mentioned previously at the end of Sec. II. We also stress that, in the case where $h$ is much larger than all other coefficients, the flow equation approximation works well even when expanded around $2 h / \omega \gg 1$, which is not what one would normally expect from a high-frequency expansion. The flow equation approach does not make the assumption at any point that $V(t)$ is small, and therefore it handles this regime more accurately.

We are particularly interested in quantum many-particle systems with a large number of degrees of freedom. We therefore compute the mismatch $E$, Eq. (42), of the timeevolution operators for a long spin chain. We plot the relative error $E$ as a function of the number of $k$-points to find out how many $k$-points are needed for a stable result. (The details on how the time-evolution operator was calculated are given in Appendix C). The plots for the Magnus expansion and for the flow equation approach are given in Fig. 7. From Fig. 7, one can see that at 256 $k$-points, the value of the relative error $E$ has stabilized. Therefore, for this model, all further plots will be done by sampling $256 k$-points.

To study the accuracy of the different approximations as a function of frequency, we choose a set of coefficients $D=0.1, J_{x}=1, J_{y}=1.1, h_{0}=1$, and $h=1$, where $J_{x}$ is fixed at unity because one may divide the Hamiltonian by $J_{x}$ to make it dimensionless. The strength of $D$ is chosen to be small since the antisymmetric exchange is often small when compared to the exchange interactions. The other values are chosen to be in a similar range. The plot of the relative error $E$ as a function of frequency $\omega$ is given in Fig. 8.

From Fig. 8, one can see that the results from the flow equation approach are valid down to much lower frequencies $\omega$. In fact, one can expect higher-order Magnus expansions to become worse at lower frequencies than the first-order Magnus expansion we plotted. This is because the optimal cutoff order of the Magnus expansion (and a number of other high-frequency expansions) shrinks with decreasing frequencies [53] unless couplings are small
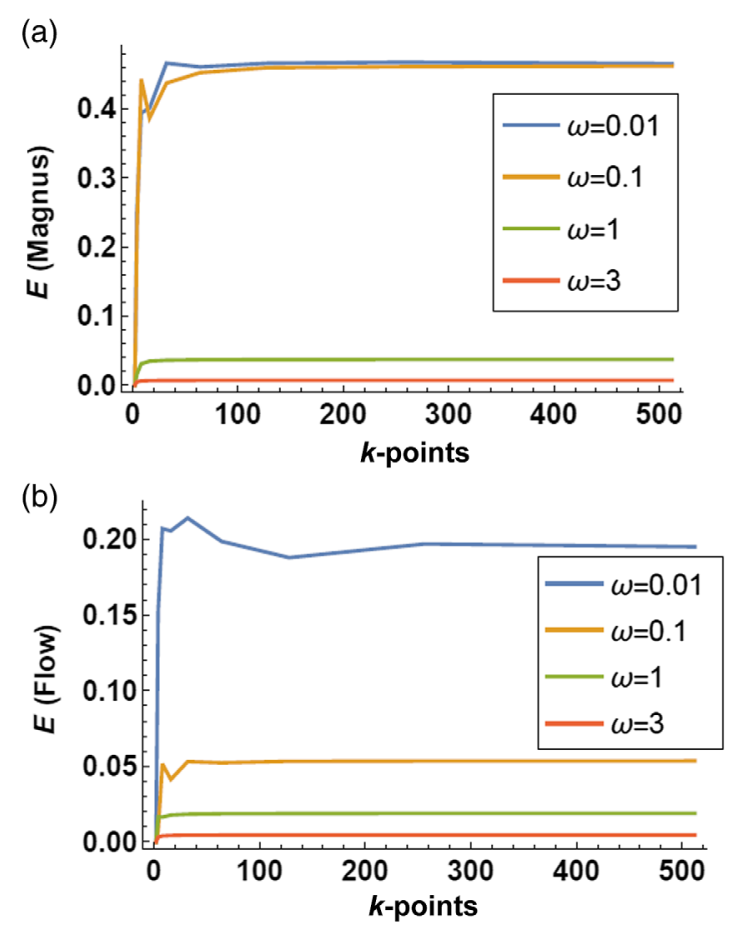

FIG. 7. Relative error, Eq. (42), of the time-evolution operator as a function of sampled $k$-points for the (a) Magnus expansion and (b) flow equation approach. Different driving frequencies $\omega=0.01, \omega=0.1, \omega=1$, and $\omega=3$ are considered. Note that the flow equation error is much smaller than the Magnus expansion error, particularly at the lowest frequencies. In both approximations, the error decreases as the frequency increases. We consider the case of $D=0.1, \quad J_{x}=1, \quad J_{y}=1.1$, $h_{0}=1$, and $h=1$.

enough to suppress this effect. It should also be noted that the stuttering (wiggles) at low frequencies seen in the plot is an effect that happens because the $U_{k}$ matrices are relatively small. For larger matrices, this effect averages out, as we see in interacting models later.

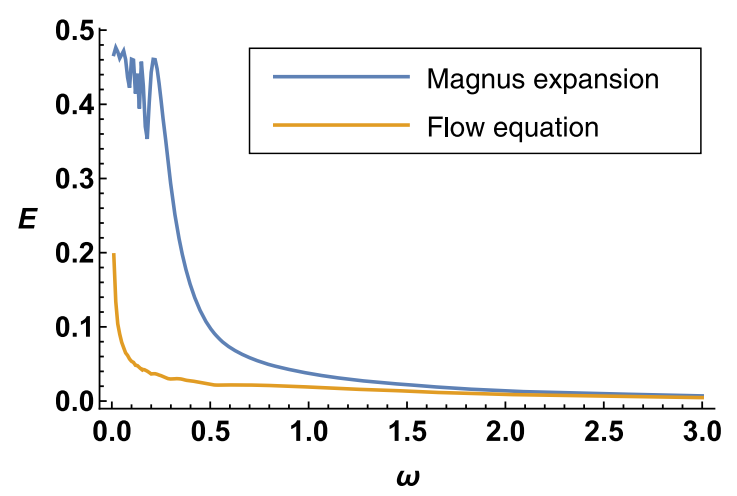

FIG. 8. Relative error $E$, Eq. (42), for $D=0.1, J_{x}=1$, $J_{y}=1.1, h_{0}=1$, and $h=1$ as a function of driving frequency $\omega$. Note how the flow equation approach outperforms the Magnus expansion, particularly at smaller $\omega$. 
(a)

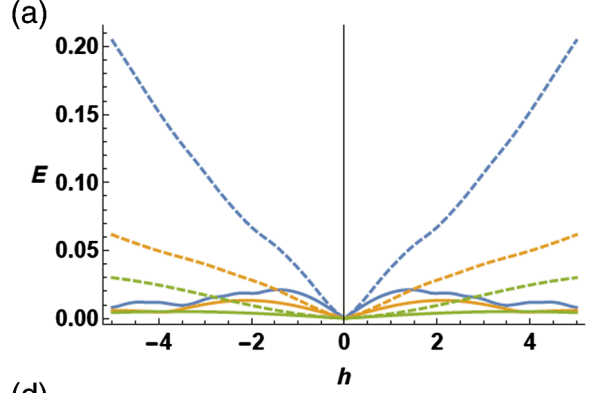

(d)

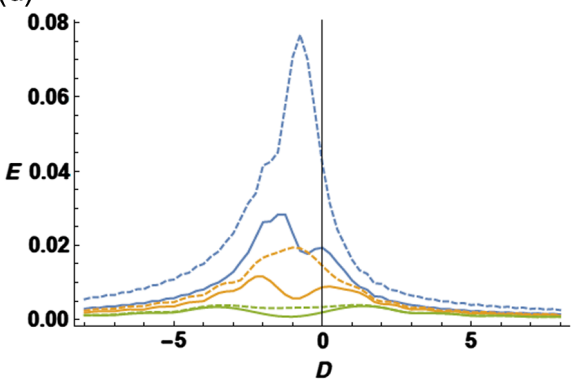

(b)

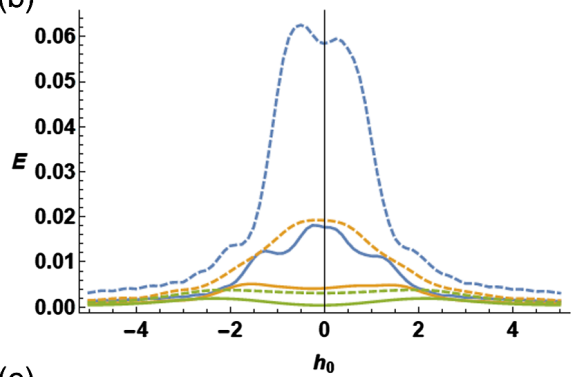

(e)

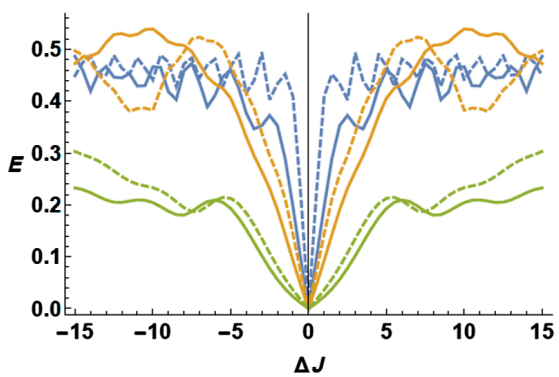

(c)

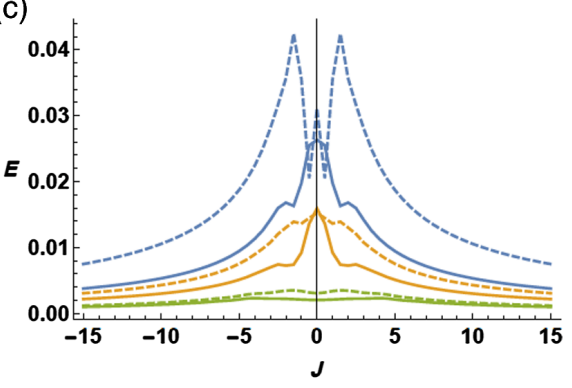

Flow equation result $\omega=1$

Magnus expansion result $\omega=1$

Flow equation result $\omega=2$

Magnus expansion result $\omega=2$

Flow equation result $\omega=4$

Magnus expansion result $\omega=4$

FIG. 9. Relative error $E$, Eq. (42), as a function of the different coupling constants in the Hamiltonian, Eq. (32). Only one coefficient is varied in each subfigure, while the ones that are not varied are fixed at values of $D=0.1, \Delta J=J_{x}-J_{y}=0.1$, $J=\left(J_{x}+J_{y} / 2\right)=1.05, h_{0}=1$, and $h=1$. In panel (a), we vary the driving magnetic field $h$, in panel (b) the static magnetic field $h_{0}$, in panel (c) the average exchange interaction $J=\left(J_{x}+J_{y} / 2\right)$, in panel (d) the antisymmetric exchange strength $D$, and in panel (e) the anisotropy of the exchange interaction $\Delta J=J_{x}-J_{y}$.

In Fig. 9, we show how well the approximation performs as a function of various couplings. From the plots, it is clear that the results obtained via the flow equation approach are generally more accurate than the results from the Magnus expansion. As expected from general arguments, we find that the approximation performs increasingly well for large values of driving $h$. We now turn to nonintegrable models.

\section{B. $J_{1}-J_{2}$ model with time-dependent magnetic field in the $x$ direction}

For this model, both the Magnus expansion and the flow equation approach yield effective Hamiltonians of the form (for a general model, the terms-quantum operatorsappearing in the effective Hamiltonians need not be the same)

$$
\begin{aligned}
H_{\text {eff }}= & \sum_{n=1}^{2} \sum_{i}\left(J_{n} S_{i}^{x} S_{i+1}^{x}+J_{n}^{y,(a)} S_{i}^{y} S_{i+n}^{y}\right. \\
& \left.+J_{n}^{z,(a)} S_{i}^{z} S_{i+n}^{z}+\Gamma_{n}^{(a)}\left(S_{i}^{z} S_{i+n}^{y}+S_{i}^{y} S_{i+n}^{z}\right)\right),
\end{aligned}
$$

where (a) labels the approximation scheme (either flow or Magnus). The details of the calculation are given in Appendix B.

It is important to note that one of the new terms, $\Gamma_{n}$, can be removed by a suitable rotation in spin space, which tells us that we went from an $X X Z$ model to an $X Y Z$ model followed by a rotation in spin space. The effective coefficients for the Magnus expansion are

$$
\begin{aligned}
J_{n}^{y, M} & =J_{n}, \\
J_{n}^{z, M} & =J_{n}^{z}, \\
\Gamma_{n}^{(M)} & =B \pi \frac{J_{n}^{z}-J_{n}}{2 \omega},
\end{aligned}
$$

and for the flow equation approach,

$$
\begin{aligned}
J_{n}^{y, M} & =\frac{1}{2}\left[\left(J_{n}^{z}+J_{n}\right)-\left(J_{n}^{z}-J_{n}\right) \operatorname{sinc}\left(\frac{2 \pi B}{\omega}\right)\right], \\
J_{n}^{z, M} & =\frac{1}{2}\left[\left(J_{n}^{z}+J_{n}\right)+\left(J_{n}^{z}-J_{n}\right) \operatorname{sinc}\left(\frac{2 \pi B}{\omega}\right)\right], \\
\Gamma_{n}^{(M)} & =\frac{\left(J_{n}^{z}-J_{n}\right) \omega \sin ^{2}\left(\frac{\pi B}{\omega}\right)}{2 \pi B} .
\end{aligned}
$$

Calculating higher orders in the Magnus expansion for this model yields extremely complicated effective Hamiltonians. The second-order Magnus expansion already gives a Hamiltonian that is a sum of 60 different operators with complicated prefactors. One tractable way to improve on the first-order Magnus expansion is via the flow equation approach. The plots in Fig. 10 illustrate the quality of the approximation for different frequencies. These results are obtained numerically using exact diagonalization for finitesize systems, as described in Appendix D.

One finds that the flow equations outperform the Magnus expansion for all frequencies. For the plot of strong driving magnetic field $h$, this is especially pronounced. There, the Magnus expansion for a large range of frequencies gives 
(a)
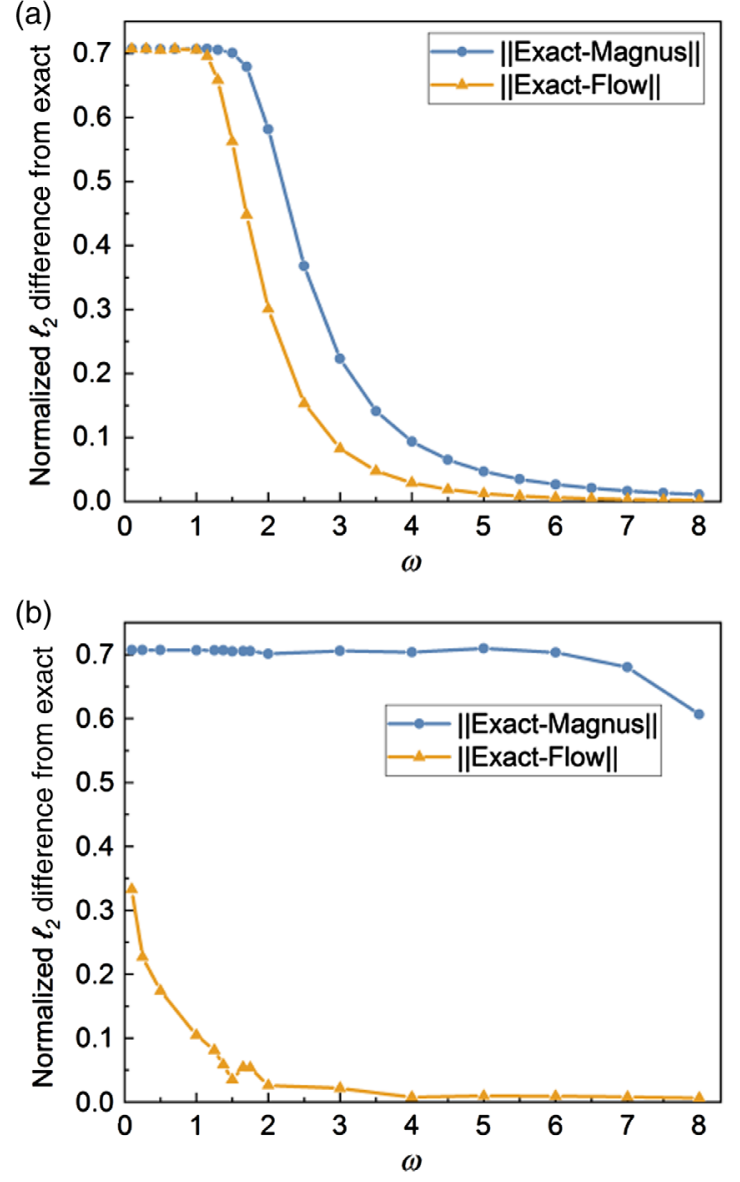

FIG. 10. Plot of the normalized $l_{2}$ distance between exact and approximate time-evolution operators for a chain of length $L=16$, and parameters $J_{1}=-0.5, J_{1}^{z}=1$, and $J_{2}=J_{2}^{z}=0$ plotted as a function of frequency for driving magnetic-field strengths (a) $B=0.5$ and (b) $B=5$.

poor results, and the flow equations generally give quite precise results.

In this case, one may also ask how well the approximation performs as a function of all the different coefficients. In Fig. 11, we show a plot for different values of the coefficients. This plot only includes the sector $k=0$ in $k$-space because it is numerically quicker and because other points in $k$-space reproduce the same results.

Similar to the previous integrable model, for this nonintegrable model, one can see that the flow equation approach outperforms the Magnus expansion for most parameters. The much higher accuracy for large values of $h$ should be emphasized. The details on how the time-evolution operator was obtained are contained in Appendix D.

\section{C. $J_{1}-J_{2}$ model with time-dependent exchange interaction}

Both the Magnus expansion and the flow equations yield an effective Hamiltonian of the form (some of the terms are zero for the Magnus case)

$$
\begin{aligned}
H_{\mathrm{eff}}= & \sum_{i} \sum_{n=1}^{2}\left\{J_{n}^{(a)} \mathbf{S}_{i}^{\perp} \cdot \mathbf{S}_{i+n}^{\perp}+J_{z, n}^{(a)} S_{i}^{z} S_{i+n}^{z}\right. \\
& +D_{n}^{(a)}\left[\mathbf{S}_{i+n+1} \times \mathbf{S}_{i+1}+\mathbf{S}_{i-n-1} \times \mathbf{S}_{i-1}\right]_{z} S_{i}^{z} \\
& \left.+Q_{n}^{(a)}\left[S_{i}^{x} S_{i+n}^{x}+S_{i}^{y} S_{i+n}^{y}\right] S_{i-1}^{z} S_{i+n+1}^{z}\right\},
\end{aligned}
$$

where $\mathbf{S}_{i}^{\perp}=\left(S_{i}^{x}, S_{i}^{y}, 0\right)$ and (a) labels the approximation scheme.

The last two terms of Eq. (51) are newly generated terms in the Hamiltonian. If $S_{i}^{z}$ has an approximately uniform orientation, the terms proportional to $D_{n}^{(a)}$ can be interpreted as different range antisymmetric exchange terms-when treating $S_{i}^{z}$ as a mean-field term. By the same token, in a mean-field approximation, the term proportional to $Q_{n}^{(a)}$ can be interpreted as an exchange term. Beyond the mean-field case, it is clear that higher-order spin interactions are generated. Such terms can lead to new physics and can drive new phases.

The coupling constants within the flow equation approach [solving Eq. (10) exactly] are given by

$$
\begin{aligned}
J_{n}^{F} & =\frac{1}{2} J_{n}\left[1+\operatorname{sinc}\left(\frac{\pi J_{1, o}^{z}}{\omega}\right)\right], \\
J_{z, 1}^{F} & =J_{1}^{z} ; \quad J_{z, 2}^{F}=J_{2}, \\
D_{n}^{F} & =\frac{J_{n} \omega}{\pi J_{1, o}^{z}}\left[\cos \left(\frac{\pi J_{1, o}^{z}}{\omega}\right)-1\right], \\
Q_{n}^{F} & =2 J_{n}\left[1-\operatorname{sinc}\left(\frac{\pi J_{1, o}^{z}}{\omega}\right)\right],
\end{aligned}
$$

and within the Magnus expansion,

$$
\begin{aligned}
& J_{n}^{M}=J_{n} ; \quad J_{z, 1}^{F}=J_{1}^{z} ; \quad J_{z, 2}^{M}=J_{2}, \\
& D_{n}^{M}=-\frac{\pi}{2 \omega} J_{n} J_{1, o}^{z} ; \quad Q_{n}^{M}=0 .
\end{aligned}
$$

While the form of the Hamiltonian in Eq. (51) is already complicated (with three- and four-spin interactions), it is worth noting that the second-order Magnus expansion would become forbiddingly complicated with a sum of over 100 operators, which makes even a numerical implementation impractical. Therefore, the result from the flow equations, while also complicated, is a significant improvement on the first-order Magnus expansion.

In Fig. 12, we plot the frequency dependence of the approximation. One finds that the flow equation result is much better in the lower-frequency regime and outperforms the Magnus approximation significantly when the external drive is relatively strong. The performance of the two approximations as a function of the different couplings is shown in the plots in Fig. 13. Consistent with the models previously discussed, the flow equation approximation performs substantially better across all parameter regimes. For this case, we made use of the QuSpin package [99] to obtain a comparison to the exact result. 
(a)

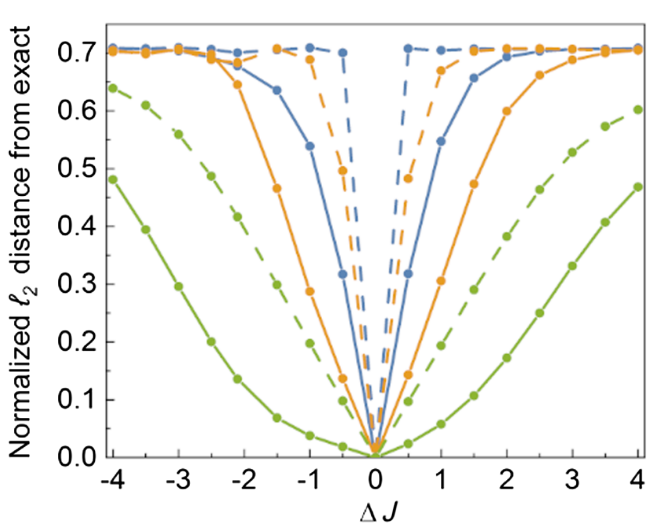

(c)

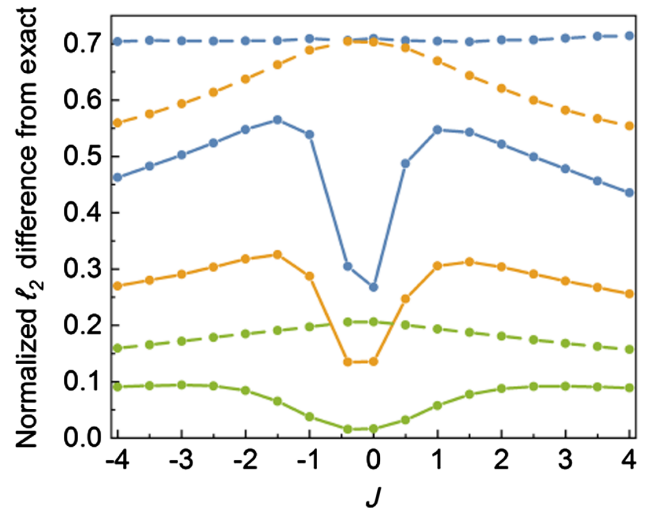

(b)

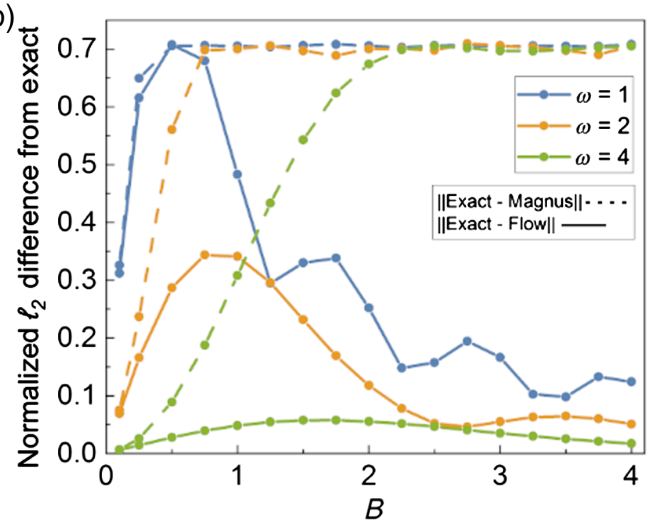

(d)

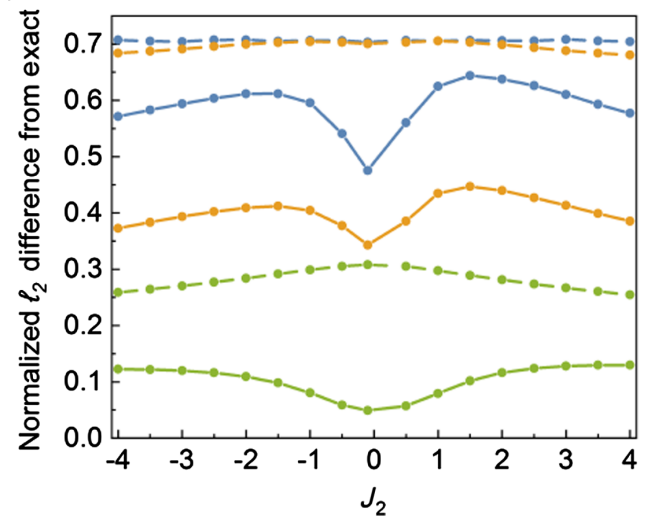

FIG. 11. Plots of the normalized $l_{2}$ distance for a chain of length $L=14$ where we vary the various coupling constants while keeping others fixed. Particularly, we are (a) varying nearest-neighbor exchange anisotropy $\Delta J=J_{1}-J_{1}^{z}$ while keeping $B=1$, average nearestneighbor exchange $J=\left(J_{1}+J_{1}^{z} / 2\right)=1$, and $J_{2}=J_{2}^{z}=0$ fixed, (b) varying $B$ while keeping $J_{1}=-0.5, J_{1}^{z}=1$, and $J_{2}=J_{2}^{z}=0$ fixed, (c) varying $J$ while keeping $\Delta J=1, B=1$, and $J_{2}=0$ fixed, and (d) varying $J_{2}$ while keeping $\Delta J=1, B=1$, and $J=1$ fixed.

\section{COMPARISON WITH RESUMMATIONS OF THE BAKER-CAMPBELL-HAUSDORFF IDENTITY}

In this section, we turn the logic around relative to the conventional Hamiltonian-evolution operator relationship.
Up to this point, we have discussed computing an effective time-independent Hamiltonian for a time-dependent problem, and we have used this effective Hamiltonian to compute the time evolution of the system. Now, we turn our attention to a situation in which the time-evolution (a)

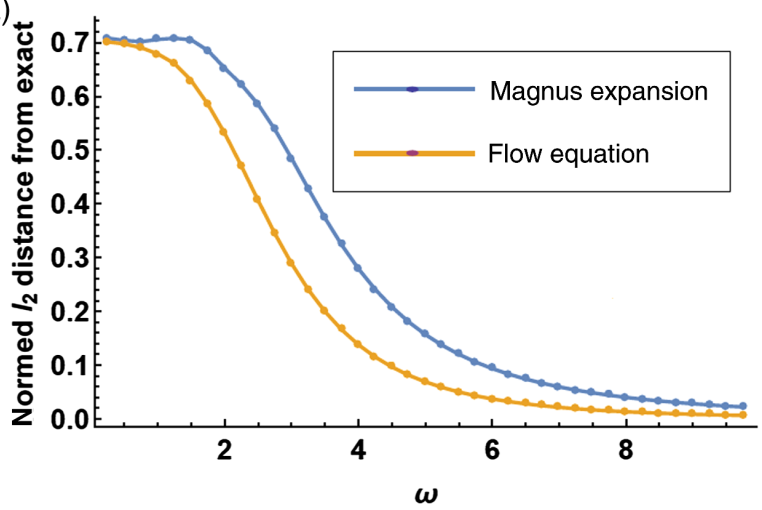

(b)

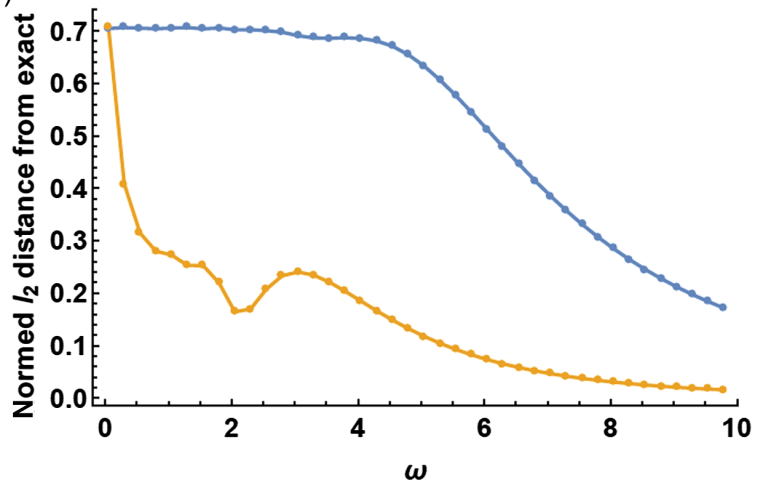

FIG. 12. Plot of the normalized $l_{2}$ distance between exact and approximate time-evolution operators for $J_{1}=1, J_{1}^{z}=2$, and $J_{2}=0.2$ plotted as a function of frequency for a spin chain with $L=14$ sites. The driving strengths of the nearest-neighbor exchange term in the $z$ direction are (a) $J_{1, o}^{z}=2$ and (b) $J_{1, o}^{z}=6$. 
(a)

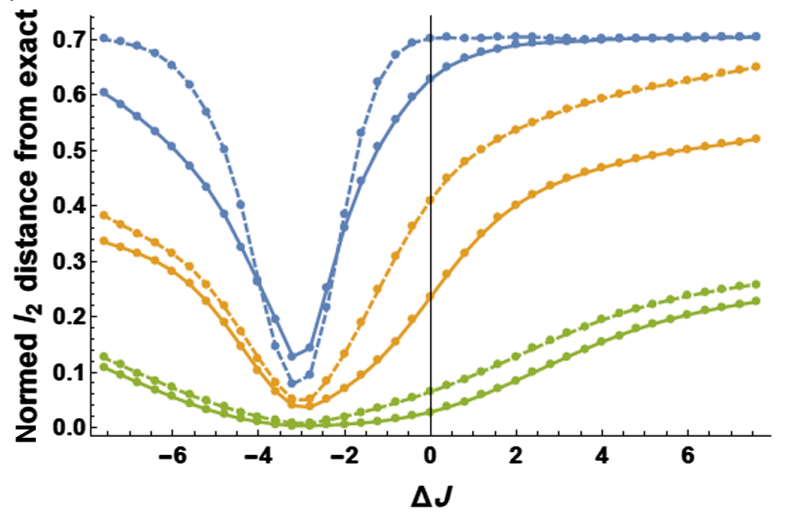

(c)

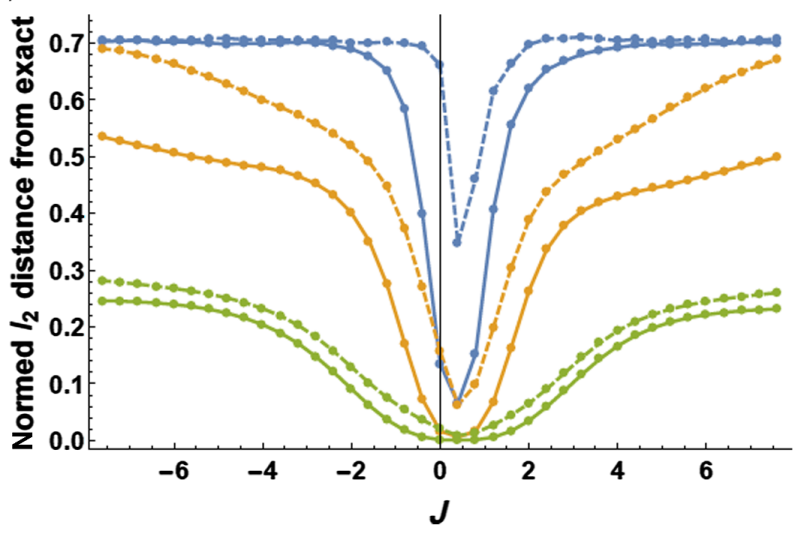

(b)

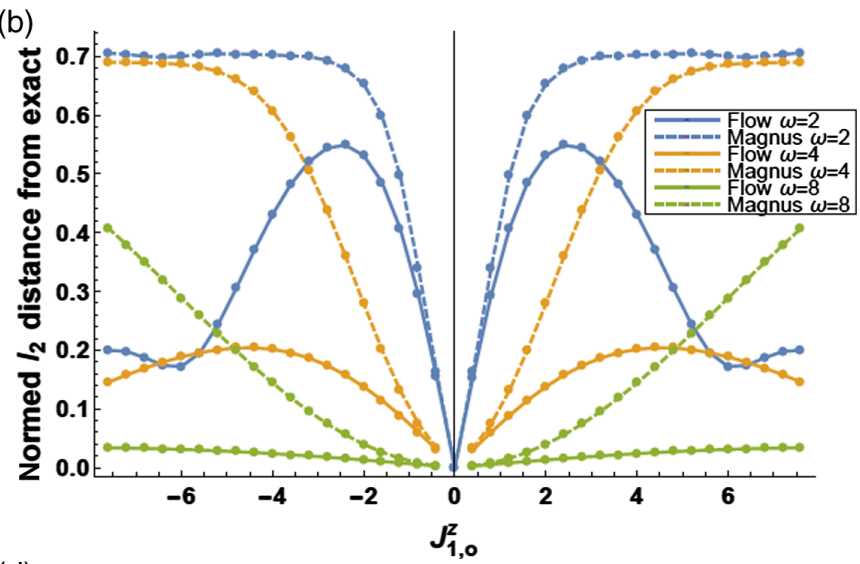

(d)

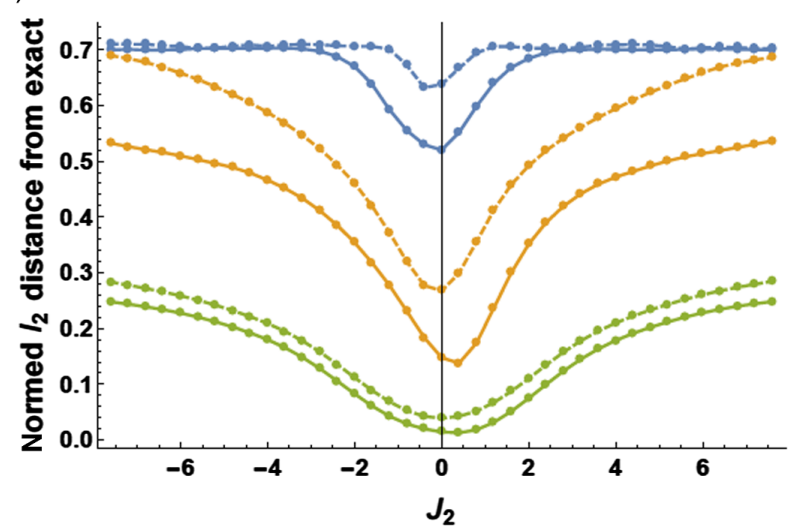

FIG. 13. Plots of the $l_{2}$ distance as a function of various coupling constants for a chain of length $L=14$. In panel (a), we vary the nearest-neighbor exchange anisotropy $\Delta J=J_{1}-J_{1}^{z}$ and keep $J_{1, o}^{z}=2, J=\frac{1}{2}\left(J_{1}+J_{1}^{z}\right)=1.5$, and $J_{2}=0.2$ fixed; in panel (b), we vary the driving strength of the exchange interaction in the $z$ direction $J_{1, o}^{z}$ and keep $\Delta J=-1, J=1.5$, and $J_{2}=0.2$ fixed; in panel (c), we vary the average nearest-neighbor exchange interaction $J$ and keep $J_{1, o}^{z}=2, \Delta J=-1$, and $J_{2}=0.2$ fixed; and in panel (d), we vary the next-nearest-neighbor exchange $J_{2}$ while keeping $J_{1, o}^{z}=2, \Delta J=-1$, and $J=1.5$ fixed.

operator is known (in our case, it takes a specific product form), and we wish to determine an optimal Hamiltonian that can be used to produce the desired time evolution. This may be useful in certain quantum computing applications, for example.

A second goal of this section is to show that our method has advantages over the rotating frame approximation. For instance, one can capture most of its features by a truncated ansatz even when an exact rotating frame approximation cannot be calculated because the effective Hamiltonian would include infinitely many long-range interacting terms. This highlights another important dimension to our flow equation approach, beyond the examples illustrating its use in earlier sections of the paper.

There has been a recent surge of interest in resummations of the Baker-Campbell-Haussdorff (BCH) identity [81]. An important evolution case where the $\mathrm{BCH}$ identity is useful is when the time-evolution operator factorizes into a product of matrix exponentials $e^{-i H_{1} t} e^{-i H_{2} t}$. This structure corresponds to multiple different Schrödinger equations. One possible correspondence is to a delta function time dependence in the Schrödinger equation. For example, the periodically kicked transverse field Ising model that is discussed in Ref. [81] consists of quenches between

$$
\begin{aligned}
& H_{1}=J \sum_{i} \sigma_{i}^{z} \sigma_{i+1}^{z}, \\
& H_{2}=\sum_{i}\left(h_{x} \sigma_{x}^{i}+h_{z} \sigma_{i}^{z}\right)
\end{aligned}
$$

and can be put into the form

$$
\begin{aligned}
H(t) & =H_{0}+V(t), \\
H_{0} & =\sum_{i}\left[J_{z} \sigma_{i}^{z} \sigma_{i+1}^{z}+h_{x} \sigma_{i}^{x}+h_{z} \sigma_{i}^{z}\right], \\
V(t) & =\sum_{i}\left[h_{x} \sigma_{i}^{x}+h_{z} \sigma_{i}^{z}\right](\delta(t)-1),
\end{aligned}
$$

where, to stay close to the notation of Ref. [81], we use Pauli operators $\sigma_{i}^{x, y, z}=2 S_{i}^{x, y, z}$ rather than the spin operators we used earlier in this work. Here, $\delta(t)$ is the Dirac delta function.

Another possibility is to rewrite the problem in terms of a Heaviside $\theta$ function as 
(a)

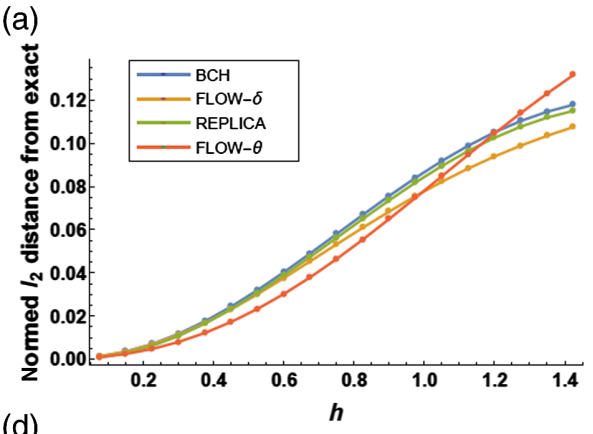

(d)

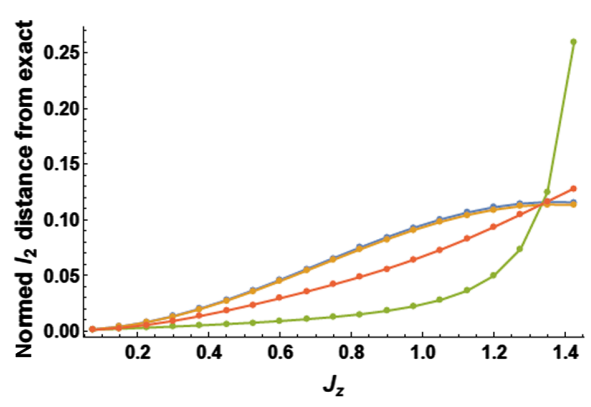

(b)

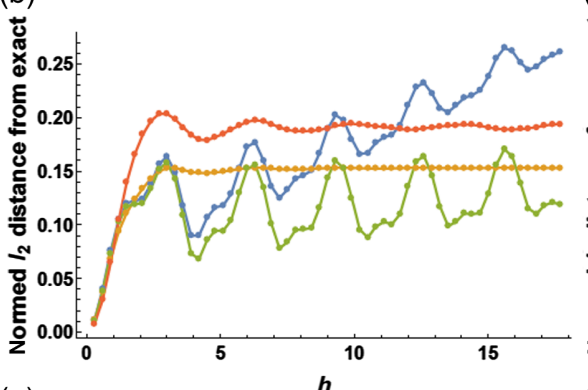

(e)

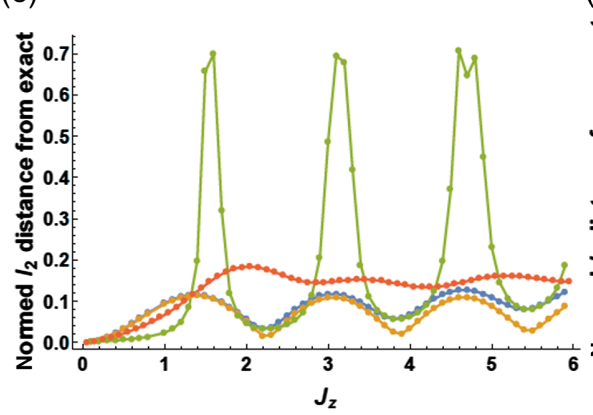

(c)

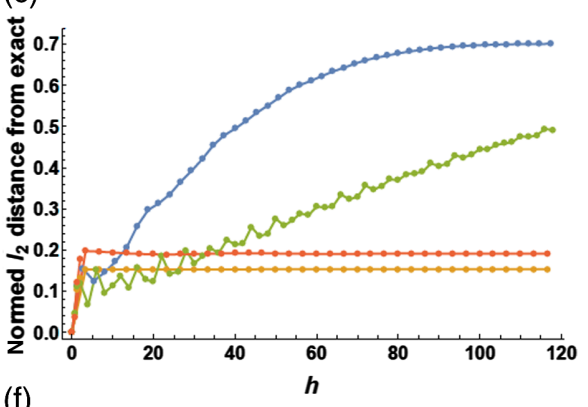

(f)

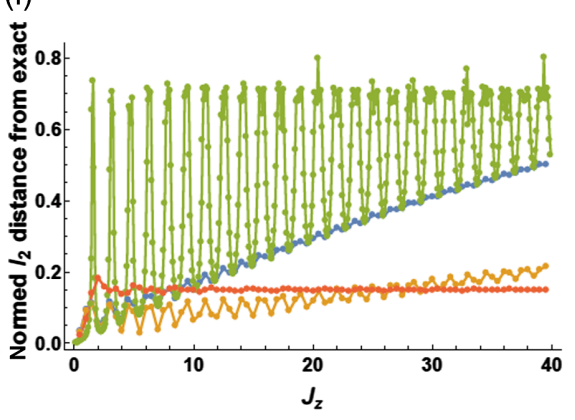

FIG. 14. Plot of the normalized $l_{2}$ distance between the exact time-evolution operator and the approximate time-evolution operator for $h_{x}=h \cos \theta, h_{z}=h \sin \theta$, and $\theta=0.643501$ using the " $\delta$ formulation" of the flow equations in orange and the "Heaviside $\theta$ formulation" in red. In plots (a)-(c), we keep $J_{z}=0.1$ fixed and plot different ranges of $h$ values, which are (a) short range $h$, (b) medium range $h$, and (c) long range $h$. Similarly, in plots (d)-(f), we keep $h=0.1$ fixed and plot different ranges of $J_{z}$ values, which are (d) short range $J_{z}$, (e) medium range $J_{z}$, and (f) long range $J_{z}$.

$$
\begin{aligned}
H(t) & =H_{0}+V(t), \\
H_{0} & =\sum_{i}\left[J_{z} \sigma_{i}^{z} \sigma_{i+1}^{z}+h_{x} \sigma_{i}^{x}+h_{z} \sigma_{i}^{z}\right], \\
V(t) & =\sum_{i}\left[h_{x} \sigma_{i}^{x}+h_{z} \sigma_{i}^{z}-J_{z} \sigma_{i}^{z} \sigma_{i+1}^{z}\right](2 \theta(t-1 / 2)-1) .
\end{aligned}
$$

Both choices lead to different flow equations and can therefore be interpreted as leading to different resummations of the BCH identity. Thus, we discuss here these two Hamiltonian choices for a given time-evolution operator. As a matter of fact, there are infinitely many ways to make a choice in the time dependence and likely one is an ideal choice. However, we will not discuss this issue of optimal choice any further. An important difference between the two formulations is that the flow equations in one case can be solved exactly, and in the other case, they require truncation. This difference allows us to assess how useful our method is in a case where the rotating frame approximation cannot be calculated exactly. This example helps illustrate the point that even when the flow equations are not solved exactly, they still give results beyond the Magnus expansion.

One finds that within the lowest order in the $\mathrm{BCH}$ expansion, the replica approximation used in Ref. [81] and our flow equation approach lead to an approximate Floquet Hamiltonian of the form

$$
\begin{aligned}
H_{\mathrm{eff}}^{(a)}= & \sum_{i}\left[C_{x}^{(a)} \sigma_{i}^{x}+C_{z}^{(a)} \sigma_{i}^{z}+C_{x x}^{(a)} \sigma_{i}^{x} \sigma_{i+1}^{x}\right. \\
& +C_{y y}^{(a)} \sigma_{i}^{y} \sigma_{i+1}^{y}+C_{z z}^{(a)} \sigma_{i}^{z} \sigma_{i+1}^{z} \\
& +C_{x y}^{(a)} \sigma_{i}^{x}\left(\sigma_{i-1}^{y}+\sigma_{i+1}^{y}\right)+C_{x z}^{(a)} \sigma_{i}^{x}\left(\sigma_{i-1}^{z}+\sigma_{i+1}^{z}\right) \\
& \left.+C_{y z}^{(a)} \sigma_{i}^{y}\left(\sigma_{i-1}^{z}+\sigma_{i+1}^{z}\right)+C_{x z z}^{(a)} \sigma_{i}^{x} \sigma_{i-1}^{z} \sigma_{i+1}^{z}\right]
\end{aligned}
$$

where $a$ labels the approximation scheme. The different approximations only differ in their coefficients (and some coefficients may be zero). The coefficients themselves offer little to illuminate our discussion. Therefore, their derivation is given in Appendix E.

In Fig. 14, we show a comparative plot for the $\delta$-type and the Heaviside-type resummations. The plots are done for spin chains of length $L=14$ to get a smooth plot. There are only small numerical differences for longer spin chains.

In the plots, one can see that the flow equation approach in Eq. (10) performs better for small values of coupling strength than the Magnus expansion-in some cases, also better than the replica expansion. For large couplings, it outperforms both.

From Fig. 14, one can see that the flow equation approach is the most reliable approximation, with the mismatch in some cases plateauing at values of around 0.1 . For those values, one is still able to capture at least qualitative features of the time evolution. Thus, the flow equation approach offers a useful numerical strategy for finding a Hamiltonian 
describing a given time evolution. This may be of practical importance in a wide variety of applications where it is difficult to determine the underlying Hamiltonian from microscopic considerations, as may be the case in various types of quantum information scenarios.

We would also like to stress that for the step-wise drive, it is not possible to calculate the exact rotating frame transformation, and therefore, a truncated ansatz for the Hamiltonian has to be employed to solve Eq. (10). One can see that this truncated ansatz performs well (red curve). It should be stressed that the truncated ansatz performs similarly to the case where an exact rotating frame approximation is possible. Our method therefore allows one to capture properties of a rotating frame approximation even when it is not possible to calculate this approximation exactly.

\section{CONCLUSIONS}

In conclusion, we have introduced an accurate "flow equation" approach to compute effective time-independent Hamiltonians, valid for finite times (which may be exponentially long) for periodically driven quantum manyparticle systems. We have demonstrated the power of the flow equation approach by illustrating how one can reach into perturbatively inaccessible frequency regimes. We have shown that the approximation generally yields an improvement over the Magnus expansion and that it can also outperform the rotating frame approximation. Furthermore, in many instances, the results from the flow equation approach also yield a practically accessible improvement on the first-order Magnus expansion, where no other method appears to be available. A straightforward application of the Magnus expansion leads to an explosion in the number of different operators that contribute to the effective Hamiltonian with coefficients that are tedious to evaluate. In our approach, one is able to truncate the number of operators contributing to the flow equations in a controlled way, which allows one to keep fewer terms but find highly accurate coefficients. We have also demonstrated that our method compares favorably to resummations of the Baker-Campbell-Hausdorff identity, illustrating that it shows its strength even in niche applications, where more powerful methods are expected. Our approach also has a wider range of applicability than standard rotating frame approximations since, even if a rotating frame approximation is impractical or not possible because the matrix exponential or the rotation of operators induced by it cannot be calculated, our method allows for a truncated ansatz that may still capture the important features of the transformation.

In summary, we hope that the demonstration of the validity of our approximate method illustrates its power and potential impact on time-dependent quantum manybody systems. The method is completely general and applicable to any form of time-dependent terms in the
Hamiltonian-be it through the potential energy, kinetic energy, or both. With the accurate, effective, timeindependent Hamiltonians that one obtains, new access is granted to potential prethermal regimes with properties not present in the equilibrium phase diagram of the original Hamiltonian. Our results also open the door to new opportunities for quantum control through Hamiltonian engineering to create the desired properties out of equilibrium. The effective Hamiltonian can be used to compute any observable over finite times through the standard formulas of statistical mechanics, in addition to accurately governing the evolution of the quantum states themselves. We hope our approach will inspire new studies that exploit its flexibility and expand the range of approximation schemes that can be employed within it. Thus, new regimes of cold atom, condensed matter, and other systems will likely be uncovered and manipulated in new ways.

\section{ACKNOWLEDGMENTS}

We gratefully acknowledge funding from Army Research Office Grant No. W911NF-14-1-0579, NSF Grant No. DMR-1507621, and NSF Materials Research Science and Engineering Center Grant No. DMR-1720595. We acknowledge the Texas Advanced Computing Center (TACC) at The University of Texas at Austin for providing computing resources that have contributed to the research results reported within this paper. www.tacc.utexas.edu G. A. F. acknowledges support from a Simons Fellowship.

\section{APPENDIX A: SPIN IN A ROTATING MAGNETIC FIELD}

The first terms of the effective Hamiltonian corresponding to Eq. (17) in a Magnus expansion are given as

$$
H_{\text {eff }} \approx\left(\begin{array}{cc}
B_{z}-\frac{B_{p}^{2}}{\omega} & -\frac{2 B_{p} B_{z}}{\omega} \\
-\frac{2 B_{p} B_{z}}{\omega} & \frac{B_{p}^{2}}{\omega}-B_{z}
\end{array}\right) .
$$

Next, we calculate the Hamiltonian in the rotating frame using Eq. (10). We make the ansatz,

$$
H(s, t)=\left(\begin{array}{cc}
B_{2}(s) & B_{0}(s)-i B_{1}(s) \\
B_{0}(s)+i B_{1}(s) & -B_{2}(s)
\end{array}\right)
$$

and find the flow equations

$$
\begin{aligned}
& \frac{d B_{2}(s)}{d s}=-\frac{2 B_{p}\left(B_{0}(s)(\cos (\omega t)-1)+B_{1}(s) \sin (\omega t)\right)}{\omega}, \\
& \frac{d B_{0}(s)}{d s}=\frac{2 B_{p} B_{2}(s)(\cos (\omega t)-1)}{\omega}-B_{p} \cos (\omega t), \\
& \frac{d B_{1}(s)}{d s}=\frac{2 B_{p} B_{2}(s) \sin (\omega t)}{\omega}-B_{p} \sin (\omega t),
\end{aligned}
$$

with initial conditions 


$$
\begin{aligned}
& B_{2}(0)=B_{z} ; \quad B_{0}(0)=B_{p} \cos (\omega t), \\
& B_{1}(0)=B_{p} \sin (\omega t) .
\end{aligned}
$$

The solutions to the flow equations at $s=1$, given our boundary conditions at $s=0$, are now given by

$$
\begin{aligned}
B_{2}= & \frac{1}{4}\left(4 B_{z}-\omega\right) \cos \left(\frac{4 B_{p} \sin \left(\frac{t \omega}{2}\right)}{\omega}\right) \\
& -B_{p} \sin \left(\frac{t \omega}{2}\right) \sin \left(\frac{4 B_{p} \sin \left(\frac{t \omega}{2}\right)}{\omega}\right)+\frac{\omega}{4} \\
B_{0}= & \frac{1}{4} \sin \left(\frac{t \omega}{2}\right)\left(\omega-4 B_{z}\right) \sin \left(\frac{4 B_{p} \sin \left(\frac{t \omega}{2}\right)}{\omega}\right) \\
& -B_{p} \sin \left(\frac{t \omega}{2}\right) \cos \left(\frac{4 B_{p} \sin ^{2}\left(\frac{t \omega}{2}\right)}{\omega}\right) \\
B_{1}= & \frac{1}{2} B_{p} \sin (t \omega) \cos \left(\frac{4 B_{p} \sin \left(\frac{t \omega}{2}\right)}{\omega}\right) \\
& -\frac{1}{4}\left(\omega-4 B_{z}\right) \cos \left(\frac{t \omega}{2}\right) \sin \left(\frac{4 B_{p} \sin \left(\frac{t \omega}{2}\right)}{\omega}\right) .
\end{aligned}
$$

After taking an average over one period, we end up with the effective time-independent Hamiltonian

$$
\begin{aligned}
H_{\mathrm{eff}} & =\left(\begin{array}{cc}
B_{2} & B_{0} \\
B_{0} & -B_{2}
\end{array}\right), \\
B_{2} & =\frac{1}{4}\left(\omega+\left(4 B_{z}-\omega\right) J_{0}\left(\frac{4 B_{p}}{\omega}\right)-4 B_{p} J_{1}\left(\frac{4 B_{p}}{\omega}\right)\right) \\
B_{0} & =B_{p} J_{2}\left(\frac{4 B_{p}}{\omega}\right)-B_{z} J_{1}\left(\frac{4 B_{p}}{\omega}\right) .
\end{aligned}
$$

\section{APPENDIX B: EFFECTIVE HAMILTONIANS}

For the lowest-order Magnus expansion, the effective Hamiltonian is

$$
H_{\text {eff }}=\frac{1}{T} \int_{0}^{t} d t\left(H(t)+i \int_{0}^{t} d t_{1}\left[H\left(t_{1}\right), H(t)\right]\right) .
$$

This result will be a reference point for our flow equation approach.

\section{Flow equation approach for the $X Y$-spin chain with antisymmetric exchange}

We find that at each flow step of Eq. (10), the Hamiltonian $H(s)$ retains the form

$$
\begin{aligned}
H(s, t)= & \sum_{i}\left(c_{1}(s, t) S_{i}^{x} S_{i+1}^{x}+c_{2}(s, t) S_{i}^{y} S_{i+1}^{y}\right. \\
& \left.+c_{3}(s, t) S_{i}^{x} S_{i+1}^{y}+c_{4}(s, t) S_{i}^{y} S_{i+1}^{x}+c_{5}(s, t) S_{i}^{z}\right),
\end{aligned}
$$

where our initial Hamiltonian, Eq. (32), tells us that we have the initial conditions

$$
\begin{aligned}
& c_{1}(0, t)=J_{x}, \\
& c_{2}(0, t)=J_{y}, \\
& c_{3}(0, t)=D, \\
& c_{4}(0, t)=-D, \\
& c_{5}(0, t)=h_{0}+h(t) .
\end{aligned}
$$

Defining $h_{I}(t):=\int_{0}^{t} d t^{\prime} h\left(t^{\prime}\right)$, we can compactly write the flow equations for the coefficients as

$$
\begin{aligned}
& \frac{d c_{1}(s, t)}{d s}=h_{I}(t)\left(c_{3}(s, t)+c_{4}(s, t)\right), \\
& \frac{d c_{2}(s, t)}{d s}=-h_{I}(t)\left(c_{3}(s, t)+c_{4}(s, t)\right), \\
& \frac{d c_{3}(s, t)}{d s}=h_{I}(t)\left(c_{2}(s, t)-c_{1}(s, t)\right), \\
& \frac{d c_{4}(s, t)}{d s}=h_{I}(t)\left(c_{2}(s, t)-c_{1}(s, t)\right), \\
& \frac{d c_{5}(s, t)}{d s}=-h(t) .
\end{aligned}
$$

The solution at $s=1$ is found as

$$
\begin{aligned}
& c_{1}(1, t)=J+\frac{\Delta J}{2} \cos \left(2 h_{I}(t)\right), \\
& c_{2}(1, t)=J-\frac{\Delta J}{2} \cos \left(2 h_{I}(t)\right), \\
& c_{3}(1, t)=D-\frac{\Delta J}{2} \sin \left(2 h_{I}(t)\right), \\
& c_{4}(1, t)=-D-\frac{\Delta J}{2} \sin \left(2 h_{I}(t)\right), \\
& c_{5}(1, t)=h_{0} .
\end{aligned}
$$

Taking the explicit form $h(t)=h \sin (\omega t)$, we can take a time average over a period of the Hamiltonian and find the approximate Hamiltonian at stroboscopic times as

$$
\begin{aligned}
H_{\mathrm{eff}}= & \sum_{i}\left(J_{x}^{(f)} S_{i}^{x} S_{i+1}^{x}+J_{y}^{(f)} S_{i}^{y} S_{i+1}^{y}+D_{+}^{(f)} S_{i}^{x} S_{i+1}^{y}\right. \\
& \left.+D_{-}^{(f)} S_{i}^{y} S_{i+1}^{x}+h_{0} S_{i}^{z}\right) \\
J_{x}^{(f)}:= & J+\frac{\Delta J}{2} \cos \left(\frac{2 h}{\omega}\right) J_{0}\left(\frac{2 h}{\omega}\right) \\
J_{y}^{(f)}:= & J-\frac{\Delta J}{2} \cos \left(\frac{2 h}{\omega}\right) J_{0}\left(\frac{2 h}{\omega}\right) \\
D_{ \pm}^{(f)}:= & \pm D-\frac{\Delta J}{2} \sin \left(\frac{2 h}{\omega}\right) J_{0}\left(\frac{2 h}{\omega}\right) .
\end{aligned}
$$


2. Flow equation approach for the $J_{1}-J_{2}$ model with time-dependent magnetic field in the $x$ direction

We find that at each flow step [using Eq. (10)], the Hamiltonian has the form

$$
\begin{aligned}
H(s, t)= & \sum_{n} \sum_{i}\left(C_{x x}^{n}(s, t) S_{i}^{x} S_{i+n}^{x}+C_{y y}^{n}(s, t) S_{i}^{y} S_{i+n}^{y}\right. \\
& \left.+C_{z z}^{n}(s, t) S_{i}^{z} S_{i+n}^{z}+C_{y z}^{n}(s, t)\left(S_{i}^{z} S_{i+n}^{y}+S_{i}^{y} S_{i+n}^{z}\right)\right) \\
& +\sum_{i} \tilde{h}(s, t) S_{i}^{z},
\end{aligned}
$$

where our initial Hamiltonian, Eq. (35), gives us the boundary conditions

$$
\begin{aligned}
C_{x x}^{n}(0, t) & =J_{n}, \\
C_{y y}^{n}(0, t) & =J_{n}, \\
C_{z z}^{n}(0, t) & =\Delta J_{n}, \\
C_{y z}^{n}(0, t) & =0, \\
\tilde{h}(0, t) & =h(t) .
\end{aligned}
$$

Defining $h_{I}(t):=\int_{0}^{t} d t^{\prime} h\left(t^{\prime}\right)$, we find that an infinitesimal step implies the flow equations

$$
\begin{aligned}
\frac{d C_{x x}^{n}(s, t)}{d s} & =0, \\
\frac{d C_{y y}^{n}(s, t)}{d s} & =2 h_{I}(t) C_{y z}^{n}(s, t), \\
\frac{d C_{z z}^{n}(s, t)}{d s} & =-2 h_{I}(t) C_{y z}^{n}(s, t), \\
\frac{d C_{y z}^{n}(s, t)}{d s} & =-h_{I}(t)\left(C_{y y}^{n}(s, t)-C_{z z}^{n}(s, t)\right), \\
\frac{d \tilde{h}(s, t)}{d s} & =-h(t) .
\end{aligned}
$$

The solution at $s=1$ is found as

$$
\begin{aligned}
C_{x x}^{n}(1, t) & =J_{n}, \\
C_{y y}^{n}(1, t) & =\frac{J_{n}}{2}\left(2+2\left(J_{n}^{z}-1\right) \sin ^{2}\left(h_{I}(t)\right)\right), \\
C_{z z}^{n}(1, t) & =\frac{J_{n}}{2}\left(2+2\left(J_{n}^{z}-1\right) \cos ^{2}\left(h_{I}(t)\right)\right), \\
C_{y z}^{n}(1, t) & =\frac{J_{n}}{2}\left(J_{n}^{z}-1\right) \sin \left(2 h_{I}(t)\right), \\
\tilde{h}(1, t) & =0 .
\end{aligned}
$$

For the special case of

$$
h(t)=B\left\{\begin{array}{ll}
1 ; & 2 n \pi<\omega t<2 n \pi+\pi \\
-1 ; & 2 n \pi+\pi<\omega t<2(n+1) \pi
\end{array} ; \quad n \in \mathbb{Z},\right.
$$

we find

$$
\begin{aligned}
H_{\text {eff }}= & \sum_{n=1}^{2} \sum_{i}\left(J_{n} S_{i}^{x} S_{i+1}^{x}+J_{n}^{y} S_{i}^{y} S_{i+n}^{y}+J_{n}^{z} S_{i}^{z} S_{i+n}^{z}\right. \\
& \left.+\Gamma_{n}\left(S_{i}^{z} S_{i+n}^{y}+S_{i}^{y} S_{i+n}^{z}\right)\right), \\
J_{n}^{y}: & \frac{J_{n}}{4}\left(2 J_{n}^{z}-\frac{\left(J_{n}^{z}-1\right) \omega \sin \left(\frac{2 \pi B}{\omega}\right)}{\pi B}+2\right), \\
J_{n}^{z}:= & \frac{J_{n}\left(\left(J_{n}^{z}-1\right) \omega \sin \left(\frac{2 \pi B}{\omega}\right)+2 \pi\left(J_{n}^{z}+1\right) B\right)}{4 \pi B}, \\
\Gamma_{n}:= & \frac{\left(J_{n}^{z}-1\right) J_{n} \omega \sin ^{2}\left(\frac{\pi B}{\omega}\right)}{2 \pi B} .
\end{aligned}
$$

\section{APPENDIX C: TIME-EVOLUTION OPERATOR FOR THE $X Y$ MODEL WITH TIME-DEPENDENT MAGNETIC FIELD IN THE $z$ DIRECTION}

The equation for the time-evolution operator in this case has the form

$$
i \partial_{t} U(t)=\sum_{k} H_{k} U(t)
$$

with $\left[H_{k}, H_{k^{\prime}}\right]=0, \forall k \neq k^{\prime}$. One may make a separation of variables ansatz

$$
U=\prod_{k} U_{k}
$$

where we assume that $\left[U_{k}, U_{k^{\prime}}\right]=0, \forall k \neq k^{\prime}$. Inserting this ansatz into Eq. $(\mathrm{C} 1)$ gives

$$
i \sum_{k}\left(\partial_{t} U_{k}\right)\left[\prod_{k^{\prime} \neq k} U_{k^{\prime}}\right]=\sum_{k} H_{k} U_{k}\left[\prod_{k^{\prime} \neq k} U_{k^{\prime}}\right] .
$$

For this equation to be fulfilled, it is sufficient that it is satisfied term by term, which yields

$$
i\left(\partial_{t} U_{k}\right)=H_{k}(t) U_{k} .
$$

In our case, generically $H_{k}(t)$ has the form

$$
H_{k}(t)=\left(c_{k}^{\dagger}, c_{-k}\right)\left(\begin{array}{ll}
H_{11}^{k}(t) & H_{12}^{k}(t) \\
H_{21}^{k}(t) & H_{22}^{k}(t)
\end{array}\right)\left(\begin{array}{c}
c_{k} \\
c_{-k}^{\dagger}
\end{array}\right) .
$$

Therefore, an ansatz for $U_{k}$ is

$$
\begin{aligned}
U_{k}(t)= & A_{0}^{k}(t)+A_{1}^{k}(t) c_{k}^{\dagger} c_{k}+A_{2}^{k}(t) c_{-k}^{\dagger} c_{-k}+A_{3}^{k}(t) c_{-k} c_{k} \\
& +A_{4}^{k}(t) c_{-k}^{\dagger} c_{k}^{\dagger}+A_{5}^{k}(t) c_{-k}^{\dagger} c_{k}^{\dagger} c_{-k} c_{k},
\end{aligned}
$$

which indeed fulfills $\left[U_{k}, U_{k^{\prime}}\right]=0, \forall k \neq k^{\prime}$. Inserting the ansatz in Eq. (C4), we find that it is consistent since no further terms appear. By equating coefficients, we find 


$$
\frac{\partial \mathbf{A}^{k}(t)}{\partial t}=i\left(\begin{array}{cccccc}
-H_{22}^{k}(t) & 0 & 0 & 0 & H_{21}^{k}(t) & 0 \\
-H_{11}^{k}(t) & -H_{11}^{k}(t)-H_{22}^{k}(t) & 0 & 0 & -H_{21}^{k}(t) & 0 \\
H_{22}^{k}(t) & 0 & 0 & 0 & -H_{21}^{k}(t) & 0 \\
-H_{21}^{k}(t) & -H_{21}^{k}(t) & -H_{21}^{k}(t) & -H_{22}^{k}(t) & 0 & H_{21}^{k}(t) \\
H_{12}^{k}(t) & 0 & 0 & 0 & -H_{11}^{k}(t) & 0 \\
0 & -H_{22}^{k}(t) & H_{11}^{k}(t) & H_{12}^{k}(t) & -H_{21}^{k}(t) & -H_{11}^{k}(t)
\end{array}\right) \mathbf{A}^{k}(t),
$$

as a linear system of equations for the coefficients $A_{i}^{k}$ from the ansatz, where we define

$$
\mathbf{A}^{k}(t)=\left(A_{1}^{k}(t), A_{2}^{k}(t), A_{3}^{k}(t), A_{4}^{k}(t), A_{5}^{k}(t)\right)^{T} .
$$

The initial conditions for a time-evolution operator in this notation are

$$
\mathbf{A}^{k}(0)=(1,0,0,0,0)^{T},
$$

where $T$ denotes the transpose (not the period of the timedependent Hamiltonian). From here, the time-evolution operator is evaluated numerically.

\section{APPENDIX D: NUMERICAL CALCULATION OF TIME-EVOLUTION OPERATORS USING EXACT DIAGONALIZATION}

To study the $J_{1}-J_{2}$ models in Eqs. (35) and (39) and their approximate counterparts in Eqs. (48) and (51), we employ exact diagonalization [100]. We note that the time evolution of a given initial state is more efficiently calculated using Krylov subspace methods, as in Ref. [57], and that DMRGbased methods are more powerful in the Floquet-MBL regime [101], being capable of reaching larger system sizes. However, here we want to compare the full timeevolution operators using the most unbiased numerical method possible. To calculate this operator for a given (exact or approximate) Hamiltonian, we write

$$
H(t)=H_{0}+V(t)
$$

where $V(t) \equiv 0$ for the time-independent effective Hamiltonians. We next "Trotterize" the problem by introducing discrete time steps $t_{j}=j T / N_{\text {steps }} \equiv j \delta t$, where $\delta t$ is chosen small enough not to affect the results.
Here, $T$ is the period of the time-dependent Hamiltonian. Then, the time-evolution operator over a period is given by

$$
U(T, 0)=\prod_{j=0}^{N_{\text {steps }}-1} U\left(t_{j+1}, t_{j}\right)
$$

where, using a second-order Trotter-Suzuki decomposition [102], we write

$$
\begin{aligned}
U(t+\delta t, t)= & \exp \left[-\frac{i \delta t}{2 \hbar} V\left(t+\frac{\delta t}{2}\right)\right] \exp \left[-i \delta t H_{0} / \hbar\right] \\
& \times \exp \left[-\frac{i \delta t}{2 \hbar} V\left(t+\frac{\delta t}{2}\right)\right]
\end{aligned}
$$

If the time dependence of $V(t)$ factors out, i.e., $V(t)=f(t) V_{0}$, the problem of calculating $U(T, 0)$ can be reduced to two matrix diagonalizations of $H_{0}$ and $V_{0}$, respectively, and numerically efficient matrix-matrix multiplications. If one of the matrices is integrable, the problem can be simplified further, as in Ref. [37], but we do not assume that here. We write $H_{0}$ and $V(t)$ in a basis implementing translational invariance and, for Eqs. (39) and (51), also magnetization conservation [100]. The full diagonalization of the two matrices is achieved using the FEAST Eigenvalue Solver [103].

\section{APPENDIX E: TRANSVERSE ISING MODEL: BCH, FLOW, AND REPLICA HAMILTONIANS}

In this section, we summarize the treatment of the transverse Ising model.

\section{Flow equations for the $\delta$-function model}

The flow equations for the $\delta$-function model, Eq. (55), in our approximation, Eq. (10), are found as 


$$
\begin{aligned}
\frac{d C_{x}^{F, \delta}(s, t)}{d s}= & -h_{x}(\delta(t)-1), \\
\frac{d C_{z}^{F, \delta}(s, t)}{d s}= & -h_{z}(\delta(t)-1), \\
\frac{d C_{x x}^{F, \delta}(s, t)}{d s}= & -4 h_{z}(t-1) C_{x y}^{F, \delta}(s, t), \\
\frac{d C_{x y}^{F,}(s, t)}{d s}= & 2(t-1)\left[h_{z}\left(C_{x x}^{F, \delta}(s, t)-C_{y y}^{F, \delta}(s, t)\right)\right. \\
& \left.-h_{x} C_{x z}^{F, \delta}(s, t)\right], \\
\frac{d C_{y y}^{F, \delta}(s, t)}{d s}= & 4(t-1)\left[h_{z} C_{x y}^{F, \delta}(s, t)-h_{x} C_{y z}^{F, \delta}(s, t)\right], \\
\frac{d C_{x z}^{F, \delta}(s, t)}{d s}= & 2(t-1)\left[h_{x} C_{x y}^{F, \delta}(s, t)-h_{z} C_{y z}^{F, \delta}(s, t)\right], \\
\frac{d C_{y z}^{F, \delta}(s, t)}{d s}= & 2(t-1)\left[h_{z} C_{x z}^{F, \delta}(s, t)+h_{x}\left(C_{y y}^{F, \delta}(s, t)\right.\right. \\
& \left.\left.-C_{z z}^{F, \delta}(s, t)\right)\right], \\
\frac{d C_{z z}^{F, \delta}(s, t)}{d s}= & 4 h_{x}(t-1) C_{y z}^{F, \delta}(s, t),
\end{aligned}
$$

with initial conditions

$$
\begin{aligned}
& C_{x}^{F, \delta}(0, t)=h_{x} \delta(t), \\
& C_{z}^{F, \delta}(0, t)=h_{z} \delta(t), \\
& C_{z z}^{F, \delta}(0, t)=J_{z}, \\
& C_{x x}^{F, \delta}(0, t)=C_{x y}^{F, \delta}(0, t)=0, \\
& C_{y y}^{F, \delta}(0, t)=C_{x z}^{F, \delta}(0, t)=C_{y z}^{F, \delta}(0, t)=0 .
\end{aligned}
$$

The solution at $s=1$ gives coefficients

$$
\begin{aligned}
C_{x / z}^{F, \delta} & =h_{x / z}, \\
C_{x x}^{F} & =\frac{h_{x}^{2} h_{z}^{2} J_{z}(12 h-8 \sin (2 h)+\sin (4 h))}{8 h^{5}}, \\
C_{x y}^{F, \delta} & =\frac{h_{x}^{2} h_{z} J_{z} \sin ^{4}(h)}{h^{4}}, \\
C_{x z}^{F, \delta} & =\frac{h_{x} h_{z} J_{z}\left(h\left(2 h_{z}^{2}-h_{x}^{2}\right)+\sin (2 h)\left(h_{x}^{2}-h_{z}^{2}\right)-\frac{h_{x}^{2} \sin (4 h)}{4}\right)}{2 h^{5}}, \\
C_{z z}^{F, \delta} & =\frac{J_{z}\left(4 h\left(h_{x}^{4}+2 h_{z}^{4}\right)+h_{x}^{4} \sin (4 h)+8 h_{x}^{2} h_{z}^{2} \sin (2 h)\right)}{8 h^{5}}, \\
C_{y z}^{F, \delta} & =\frac{h_{x} J_{z} \sin ^{2}(h)\left(h_{x}^{2} \cos (2 h)+h_{x}^{2}+2 h_{z}^{2}\right)}{2 h^{4}}, \\
C_{y y}^{F, \delta} & =-\frac{h_{x}^{2} J_{z}(\sin (4 h)-4 h)}{8 h^{3}}, \\
C_{x z z}^{F, \delta} & =0 .
\end{aligned}
$$

\section{Flow equations for the Heaviside $\boldsymbol{\theta}$-function model}

The flow equations for the Heaviside $\theta$-function model, Eq. (56), in our approximation, Eq. (10), are found to generate an infinite amount of terms. This finding means that an exact solution of Eq. (10) is impossible; in turn, it also means that a rotating frame approximation is impossible because matrix exponentials, and also the rotation of operators induced by it, cannot be calculated. Our method allows us to truncate terms and therefore find an approximate rotating frame transformation. The terms that appear in Eq. (57) are generated quickly when using an ansatz that starts with the form of the original Hamiltonian and subsequently adding the new terms that appear to that ansatz. This result motivates one to include as many terms from the Hamiltonian Eq. (57) as possible while still allowing for a compact analytical result. We choose the ansatz Hamiltonian

$$
\begin{aligned}
H_{\text {Ansatz }}^{F, \theta}(s)= & \sum_{i}\left[C_{x}^{F, \theta} \sigma_{i}^{x}+C_{z}^{F, \theta} \sigma_{i}^{z}+C_{y y}^{F, \theta} \sigma_{i}^{y} \sigma_{i+1}^{y}\right. \\
& +C_{z z}^{F, \theta} \sigma_{i}^{z} \sigma_{i+1}^{z}+C_{x z}^{F, \theta}\left(\sigma_{i}^{x} \sigma_{i-1}^{z}+\sigma_{i}^{x} \sigma_{i+1}^{z}\right) \\
& +C_{y z}^{F, \theta}\left(\sigma_{i}^{y} \sigma_{i-1}^{z}+\sigma_{i}^{y} \sigma_{i+1}^{z}\right) \\
& \left.+C_{x z z}^{F, \theta} \sigma_{i}^{x} \sigma_{i-1}^{z} \sigma_{i+1}^{z}\right] .
\end{aligned}
$$

The flow equations, Eq. (10), give us the following equations for the coefficients:

$$
\begin{aligned}
\frac{d C_{x}^{F, \theta}(s, t)}{d s}= & -\left(4 J_{z} C_{y z}^{F, \theta}(s, t) f_{I}(t)+h_{x} f(t)\right), \\
\frac{d C_{z}^{F, \theta}(s, t)}{d s}= & -h_{z} f(t), \\
\frac{d C_{y y}^{F, \theta}(s, t)}{d s}= & 4 h_{x} C_{y z}^{F, \theta}(s, t) f_{I}(t), \\
\frac{d C_{x z}^{F, \theta}(s, t)}{d s}= & 2 h_{z} C_{y z}^{F, \theta}(s, t) f_{I}(t), \\
\frac{d C_{y z}^{F, \theta}(s, t)}{d s}= & 2 f_{I}(t)\left(J_{z} C_{x}^{F, \theta}(s, t)-h_{z} C_{x z}^{F, \theta}(s, t),\right. \\
& \left.+J_{z} C_{x z z}^{F, \theta}(s, t)-h_{x} C_{y y}^{F, \theta}(s, t)+h_{x} C_{z z}^{F, \theta}(s, t)\right), \\
\frac{d C_{z z}^{F, \theta}(s, t)}{d s}= & \left(J_{z} f(t)-4 h_{x} C_{y z}^{F, \theta}(s, t) f_{I}(t)\right), \\
\frac{d C_{x z z}^{F, \theta}(s, t)}{d s}= & -4 J_{z} C_{y z}^{F, \theta}(s, t) f_{I}(t),
\end{aligned}
$$

where $f(t)=\theta\left(t-\frac{1}{2}\right), f_{I}(t)=t+(1-2 t) \theta\left(t-\frac{1}{2}\right)$, and $\theta$ is the Heaviside function. 
The initial conditions are

$$
\begin{aligned}
& C_{x}^{F, \theta}(0, t)=h_{x}(f(t)+1), \\
& C_{z}^{F, \theta}(0, t)=h_{z}(f(t)+1), \\
& C_{z z}^{F, \theta}(0, t)=-J_{z}(f(t)-1), \\
& C_{x x}^{F, \theta}(0, t)=C_{x y}^{F, \theta}(0)=0, \\
& C_{y y}^{F, \theta}(0, t)=C_{x z}^{F, \theta}(0, t)=C_{y z}^{F, \theta}(0, t)=0 .
\end{aligned}
$$

The solution at $s=1$ implies that the coefficients are

$$
\begin{aligned}
& C_{x}^{F, \theta}=h_{x}-\frac{4 h_{x} J_{z}^{2}}{\gamma^{2}}\left(1-\frac{\sin (\gamma)}{\gamma}\right), \\
& C_{z}^{F, \theta}=h_{z}, \\
& C_{x x}^{F}=C_{x y}^{F, \theta}=0, \\
& C_{x z}^{F, \theta}=\frac{2 h_{x} h_{z} J_{z}}{\gamma^{2}}\left(1-\frac{\sin (\gamma)}{\gamma}\right), \\
& C_{z z}^{F, \theta}=J_{z}-\frac{4 h_{x}^{2} J_{z}}{\gamma^{2}}\left(1-\frac{\sin (\gamma)}{\gamma}\right), \\
& C_{y z}^{F, \theta}=\frac{2 h_{x} J_{z}}{\gamma^{2}}(1-\cos (\gamma)), \\
& C_{y y}^{F, \theta}=\frac{4 h_{x}^{2} J_{z}}{\gamma^{2}}\left(1-\frac{\sin (\gamma)}{\gamma}\right), \\
& C_{x z z}^{F, \theta}=\frac{8 h_{x} J_{z}^{2}}{\gamma^{2}}\left(\frac{\sin (\gamma)}{\gamma}-1\right),
\end{aligned}
$$

where $\gamma=\sqrt{4 h_{x}^{2}+h_{z}^{2}+4 J_{z}^{2}}$.

\section{Result for the $\mathbf{B C H}$ identity}

For the $\mathrm{BCH}$ identity, one finds the coefficients

$$
\begin{aligned}
& C_{x}^{\mathrm{BCH}}=h_{x}, \\
& C_{z}^{\mathrm{BCH}}=h_{z}, \\
& C_{z z}^{\mathrm{BCH}}=J_{z}, \\
& C_{y z}^{\mathrm{BCH}}=h_{x} J_{z}, \\
& C_{x x}^{\mathrm{BCH}}=C_{x y}^{\mathrm{BCH}}=C_{x z}^{\mathrm{BCH}}=C_{y y}^{\mathrm{BCH}}=C_{x z z}^{\mathrm{BCH}}=0 .
\end{aligned}
$$

\section{Result for the replica approximation}

The coefficients for the replica case were taken from Ref. [81] as

$$
\begin{aligned}
C_{x}^{R} & =h_{x}\left(J_{z} \cot \left(2 J_{z}\right)+\frac{1}{2}\right), \\
C_{z}^{R} & =h_{z}, \\
C_{z z}^{R} & =J_{z}, \\
C_{y z}^{R} & =\frac{1}{2} h_{x} J_{z}, \\
C_{x z z}^{R} & =h_{x}\left(J_{z} \cot \left(2 J_{z}\right)-\frac{1}{2}\right), \\
C_{x x}^{R} & =C_{x y}^{R}=C_{x z}^{R}=C_{y y}^{R}=0 .
\end{aligned}
$$

[1] A. Polkovnikov, K. Sengupta, A. Silva, and M. Vengalattore, Colloquium: Nonequilibrium Dynamics of Closed Interacting Quantum Systems, Rev. Mod. Phys. 83, 863 (2011).

[2] A. Eckardt, Colloquium: Atomic Quantum Gases in Periodically Driven Optical Lattices, Rev. Mod. Phys. 89, 011004 (2017).

[3] I. Bloch, J. Dalibard, and W. Zwerger, Many-Body Physics with Ultracold Gases, Rev. Mod. Phys. 80, 885 (2008).

[4] J. Dalibard, F. Gerbier, G. Juzeliūnas, and P. Öhberg, Colloquium: Artificial Gauge Potentials for Neutral Atoms, Rev. Mod. Phys. 83, 1523 (2011).

[5] D. N. Basov, R. D. Averitt, and D. Hsieh, Towards Properties on Demand in Quantum Materials, Nat. Mat. 16, 1077 (2017).

[6] J. Zhang and R. D. Averitt, Dynamics and Control in Complex Transition Metal Oxides, Annu. Rev. Mater. Res. 44, 19 (2014).

[7] D. N. Basov, R. D. Averitt, D. van der Marel, M. Dressel, and K. Haule, Electrodynamics of Correlated Electron Materials, Rev. Mod. Phys. 83, 471 (2011).

[8] C. Giannetti, M. Capone, D. Fausti, M. Fabrizio, F. Parmigiani, and D. Mihailovic, Ultrafast Optical Spectroscopy of Strongly Correlated Materials and High-Temperature Superconductors: A Non-equilibrium Approach, Adv. Phys. 65, 58 (2016).

[9] M. Gandolfi, G. L. Celardo, F. Borgonovi, G. Ferrini, A. Avella, F. Banfi, and C. Giannetti, Emergent Ultrafast Phenomena in Correlated Oxides and Heterostructures, Phys. Scr. 92, 034004 (2017).

[10] J. H. Mentink and M. Eckstein, Ultrafast Quenching of the Exchange Interaction in a Mott Insulator, Phys. Rev. Lett. 113, 057201 (2014).

[11] J. H. Mentink, K. Balzer, and M. Eckstein, Ultrafast and Reversible Control of the Exchange Interaction in Mott Insulators, Nat. Commun. 6, 6708 (2015).

[12] D. Fausti, R. I. Tobey, N. Dean, S. Kaiser, A. Dienst, M. C. Hoffmann, S. Pyon, T. Takayama, H. Takagi, and A. Cavalleri, Light-Induced Superconductivity in a StripeOrdered Cuprate, Science 331, 189 (2011).

[13] A. Cavalleri, Photo-Induced Superconductivity, Contemp. Phys. 59, 31 (2018). 
[14] L. Stojchevska, I. Vaskivskyi, T. Mertelj, P. Kusar, D. Svetin, S. Brazovskii, and D. Mihailovic, Ultrafast Switching to a Stable Hidden Quantum State in an Electronic Crystal, Science 344, 177 (2014).

[15] Y. A. Gerasimenko, I. Vaskivskyi, and D. Mihailovic, Long Range Electronic Order in a Metastable State Created by Ultrafast Topological Transformation, arXiv:1704 .08149 .

[16] J. Zhang, P. W. Hess, A. Kyprianidis, P. Becker, A. Lee, J. Smith, G. Pagano, I.-D. Potirniche, A. C. Potter, A. Vishwanath, N. Y. Yao, and C. Monroe, Observation of a Discrete Time Crystal, Nature (London) 543, 217 (2017).

[17] S. Choi, J. Choi, R. Landig, G. Kucsko, H. Zhou, J. Isoya, F. Jelezko, S. Onoda, H. Sumiya, V. Khemani, C. von Keyserlingk, N. Y. Yao, E. Demler, and M. D. Lukin, Observation of Discrete Time-Crystalline Order in a Disordered Dipolar Many-Body System, Nature (London) 543, 221 (2017).

[18] A. C. Potter, T. Morimoto, and A. Vishwanath, Classification of Interacting Topological Floquet Phases in One Dimension, Phys. Rev. X 6, 041001 (2016).

[19] V. Khemani, A. Lazarides, R. Moessner, and S. L. Sondhi, Phase Structure of Driven Quantum Systems, Phys. Rev. Lett. 116, 250401 (2016).

[20] M. Bukov, L. D'Alessio, and A. Polkovnikov, Universal High-Frequency Behavior of Periodically Driven Systems: From Dynamical Stabilization to Floquet Engineering, Adv. Phys. 64, 139 (2015).

[21] J. H. Shirley, Solution of the Schrödinger Equation with a Hamiltonian Periodic in Time, Phys. Rev. 138, B979 (1965).

[22] R. Moessner and S. L. Sondhi, Equilibration and Order in Quantum Floquet Matter, Nat. Phys. 13, 424 (2017).

[23] A. Lazarides, A. Das, and R. Moessner, Periodic Thermodynamics of Isolated Quantum Systems, Phys. Rev. Lett. 112, 150401 (2014).

[24] W. Berdanier, M. Kolodrubetz, R. Vasseur, and J. E. Moore, Floquet Dynamics of Boundary-Driven Systems at Criticality, Phys. Rev. Lett. 118, 260602 (2017).

[25] X. Wen and J.-Q. Wu, Floquet Conformal Field Theory, arXiv:1805.00031.

[26] P. Ponte, A. Chandran, Z. Papi, and D. A. Abanin, Periodically Driven Ergodic and Many-Body Localized Quantum Systems, Ann. Phys. (Amsterdam) 353, 196 (2015).

[27] P. Ponte, Z. Papić, F. Huveneers, and D. A. Abanin, ManyBody Localization in Periodically Driven Systems, Phys. Rev. Lett. 114, 140401 (2015).

[28] A. Lazarides, A. Das, and R. Moessner, Equilibrium States of Generic Quantum Systems Subject to Periodic Driving, Phys. Rev. E 90, 012110 (2014).

[29] Y. H. Wang, H. Steinberg, P. Jarillo-Herrero, and N. Gedik, Observation of Floquet-Bloch States on the Surface of a Topological Insulator, Science 342, 453 (2013).

[30] L. Jiang, T. Kitagawa, J. Alicea, A. R. Akhmerov, D. Pekker, G. Refael, J. I. Cirac, E. Demler, M. D. Lukin, and P. Zoller, Majorana Fermions in Equilibrium and in Driven Cold-Atom Quantum Wires, Phys. Rev. Lett. 106, 220402 (2011).
[31] P. Molignini, E. van Nieuwenburg, and R. Chitra, Sensing Floquet-Majorana Fermions via Heat Transfer, Phys. Rev. B 96, 125144 (2017).

[32] K. Jiménez-García, L. J. LeBlanc, R. A. Williams, M. C. Beeler, C. Qu, M. Gong, C. Zhang, and I. B. Spielman, Tunable Spin-Orbit Coupling via Strong Driving in Ultracold-Atom Systems, Phys. Rev. Lett. 114, 125301 (2015).

[33] J. Klinovaja, P. Stano, and D. Loss, Topological Floquet Phases in Driven Coupled Rashba Nanowires, Phys. Rev. Lett. 116, 176401 (2016).

[34] L. Du, X. Zhou, and G. A. Fiete, Quadratic Band Touching Points and Flat Bands in Two-Dimensional Topological Floquet Systems, Phys. Rev. B 95, 035136 (2017).

[35] Q. Chen, L. Du, and G. A. Fiete, Floquet Band Structure of a Semi-Dirac System, Phys. Rev. B 97, 035422 (2018).

[36] P. Titum, E. Berg, M. S. Rudner, G. Refael, and N. H. Lindner, Anomalous Floquet-Anderson Insulator as a Nonadiabatic Quantized Charge Pump, Phys. Rev. X 6 , 021013 (2016).

[37] L. D'Alessio and M. Rigol, Dynamical Preparation of Floquet Chern Insulators, Nat. Commun. 6, 8336 (2015).

[38] I. Martin, G. Refael, and B. Halperin, Topological Frequency Conversion in Strongly Driven Quantum Systems, Phys. Rev. X 7, 041008 (2017).

[39] T. Oka and H. Aoki, Photovoltaic Hall Effect in Graphene, Phys. Rev. B 79, 081406(R) (2009).

[40] N. H. Lindner, G. Refael, and V. Galitski, Floquet Topological Insulator in Semiconductor Quantum Wells, Nat. Phys. 7, 490 (2011).

[41] T. Kitagawa, T. Oka, A. Brataas, L. Fu, and E. Demler, Transport Properties of Nonequilibrium Systems under the Application of Light: Photoinduced Quantum Hall Insulators without Landau Levels, Phys. Rev. B 84, 235108 (2011).

[42] Z. Gu, H. A. Fertig, D. P. Arovas, and A. Auerbach, Floquet Spectrum and Transport through an Irradiated Graphene Ribbon, Phys. Rev. Lett. 107, 216601 (2011).

[43] M. S. Rudner, N. H. Lindner, E. Berg, and M. Levin, Anomalous Edge States and the Bulk-Edge Correspondence for Periodically Driven Two-Dimensional Systems, Phys. Rev. X 3, 031005 (2013).

[44] G. Usaj, P. M. Perez-Piskunow, L. E. F. F. Torres, and C. A. Balseiro, Irradiated Graphene as a Tunable Floquet Topological Insulator, Phys. Rev. B 90, 115423 (2014).

[45] A. Zenesini, H. Lignier, D. Ciampini, O. Morsch, and E. Arimondo, Coherent Control of Dressed Matter Waves, Phys. Rev. Lett. 102, 100403 (2009).

[46] A. Eckardt, C. Weiss, and M. Holthaus, SuperfluidInsulator Transition in a Periodically Driven Optical Lattice, Phys. Rev. Lett. 95, 260404 (2005).

[47] N. Y. Yao, A. C. Potter, I.-D. Potirniche, and A. Vishwanath, Discrete Time Crystals: Rigidity, Criticality, and Realizations, Phys. Rev. Lett. 118, 030401 (2017).

[48] I.-D. Potirniche, A. C. Potter, M. Schleier-Smith, A. Vishwanath, and N. Y. Yao, Floquet Symmetry-Protected Topological Phases in Cold-Atom Systems, Phys. Rev. Lett. 119, 123601 (2017).

[49] A. Lerose, J. Marino, A. Gambassi, and A. Silva, Quantum Many-Body Kapitza Phases of Periodically Driven Spin Systems," arXiv:1803.04490. 
[50] J. Rehn, A. Lazarides, F. Pollmann, and R. Moessner, How Periodic Driving Heats a Disordered Quantum Spin Chain, Phys. Rev. B 94, 020201(R) (2016).

[51] L. D'Alessio and M. Rigol, Long-Time Behavior of Isolated Periodically Driven Interacting Lattice Systems, Phys. Rev. X 4, 041048 (2014).

[52] D. A. Abanin, W. De Roeck, and F. Huveneers, Exponentially Slow Heating in Periodically Driven Many-Body Systems, Phys. Rev. Lett. 115, 256803 (2015).

[53] D. A. Abanin, W. De Roeck, W. W. Ho, and F. Huveneers, Effective Hamiltonians, Prethermalization, and Slow Energy Absorption in Periodically Driven Many-Body Systems, Phys. Rev. B 95, 014112 (2017).

[54] T. Mori, T. Kuwahara, and K. Saito, Rigorous Bound on Energy Absorption and Generic Relaxation in Periodically Driven Quantum Systems, Phys. Rev. Lett. 116, 120401 (2016).

[55] D. V. Else, B. Bauer, and C. Nayak, Prethermal Phases of Matter Protected by Time-Translation Symmetry, Phys. Rev. X 7, 011026 (2017).

[56] E. Canovi, M. Kollar, and M. Eckstein, Stroboscopic Prethermalization in Weakly Interacting Periodically Driven Systems, Phys. Rev. E 93, 012130 (2016).

[57] F. Machado, G. D. Meyer, D. V. Else, C. Nayak, and N. Y. Yao, Exponentially Slow Heating in Short and LongRange Interacting Floquet Systems, arXiv:1708.01620.

[58] S. A. Weidinger and M. Knap, Floquet Prethermalization and Regimes of Heating in a Periodically Driven, Interacting Quantum System, Sci. Rep. 7, 45382 (2017).

[59] T. Kuwahara, T. Mori, and K. Saito, Floquet-Magnus Theory and Generic Transient Dynamics in Periodically Driven Many-Body Quantum Systems, Ann. Phys. (Amsterdam) 367, 96 (2016).

[60] M. Heyl, A. Polkovnikov, and S. Kehrein, Dynamical Quantum Phase Transitions in the Transverse-Field Ising Model, Phys. Rev. Lett. 110, 135704 (2013).

[61] P. Jurcevic, H. Shen, P. Hauke, C. Maier, T. Brydges, C. Hempel, B. P. Lanyon, M. Heyl, R. Blatt, and C. F. Roos, Direct Observation of Dynamical Quantum Phase Transitions in an Interacting Many-Body System, Phys. Rev. Lett. 119, 080501 (2017).

[62] N. Fläschner, D. Vogel, M. Tarnowski, B. S. Rem, D.-S. Lühmann, M. Heyl, J. C. Budich, L. Mathey, K. Sengstock, and C. Weitenberg, Observation of a Dynamical Topological Phase Transition, Nat. Phys. 14, 265 (2018).

[63] L. Du and G. A. Fiete, Dynamical Recovery of $S U(2)$ Symmetry in the Mass-Quenched Hubbard Model, Phys. Rev. B 97, 085152 (2018).

[64] Yu.-A. Chen, S. Nascimbène, M. Aidelsburger, M. Atala, S. Trotzky, and I. Bloch, Controlling Correlated Tunneling and Superexchange Interactions with ac-Driven Optical Lattices, Phys. Rev. Lett. 107, 210405 (2011).

[65] C. Dutreix and M. I. Katsnelson, Dynamical Control of Electron-Phonon Interactions with High-Frequency Light, Phys. Rev. B 95, 024306 (2017).

[66] M. Bukov, S. Gopalakrishnan, M. Knap, and E. Demler, Prethermal Floquet Steady States and Instabilities in the Periodically Driven, Weakly Interacting Bose-Hubbard Model, Phys. Rev. Lett. 115, 205301 (2015).
[67] M. Bukov, M. Kolodrubetz, and A. Polkovnikov, Schrieffer-Wolff Transformation for Periodically Driven Systems: Strongly Correlated Systems with Artificial Gauge Fields, Phys. Rev. Lett. 116, 125301 (2016).

[68] F. Peronaci, M. Schiró, and O. Parcollet, Resonant Thermalization of Periodically Driven Strongly Correlated Electrons, Phys. Rev. Lett. 120, 197601 (2018).

[69] N. H. Lindner, E. Berg, and M. S. Rudner, Universal Chiral Quasisteady States in Periodically Driven ManyBody Systems, Phys. Rev. X 7, 011018 (2017).

[70] K. Plekhanov, G. Roux, and K. Le Hur, Floquet Engineering of Haldane Chern Insulators and Chiral Bosonic Phase Transitions, Phys. Rev. B 95, 045102 (2017).

[71] S. Blanes, F. Casas, J. A. Oteo, and J. Ros, The Magnus Expansion and Some of Its Applications, Phys. Rep. 470, 151 (2009).

[72] E. B. Fel'dman, On the Convergence of the Magnus Expansion for Spin Systems in Periodic Magnetic Fields, Phys. Lett. A 104, 479 (1984).

[73] W. Magnus, On the Exponential Solution of Differential Equations for a Linear Operator, Commun. Pure Appl. Math. 7, 649 (1954).

[74] S. Rahav, I. Gilary, and S. Fishman, Effective Hamiltonians for Periodically Driven Systems, Phys. Rev. A 68, 013820 (2003).

[75] N. Goldman and J. Dalibard, Periodically Driven Quantum Systems: Effective Hamiltonians and Engineered Gauge Fields, Phys. Rev. X 4, 031027 (2014).

[76] A. P. Itin and M. I. Katsnelson, Effective Hamiltonians for Rapidly Driven Many-Body Lattice Systems: Induced Exchange Interactions and Density-Dependent Hoppings, Phys. Rev. Lett. 115, 075301 (2015).

[77] A. Eckardt and E. Anisimovas, High-Frequency Approximation for Periodically Driven Quantum Systems from a Floquet-Space Perspective, New J. Phys. 17, 093039 (2015).

[78] T. Mikami, S. Kitamura, K. Yasuda, N. Tsuji, T. Oka, and H. Aoki, Brillouin-Wigner Theory for High-Frequency Expansion in Periodically Driven Systems: Application to Floquet Topological Insulators, Phys. Rev. B 93, 144307 (2016).

[79] P. Mohan, R. Saxena, A. Kundu, and S. Rao, BrillouinWigner Theory for Floquet Topological Phase Transitions in Spin-Orbit-Coupled Materials, Phys. Rev. B 94, 235419 (2016).

[80] M. M. Maricq, Application of Average Hamiltonian Theory to the NMR of Solids, Phys. Rev. B 25, 6622 (1982).

[81] S. Vajna, K. Klobas, T. Prosen, and A. Polkovnikov, Replica Resummation of the Baker-Campbell-Hausdorff Series, Phys. Rev. Lett. 120, 200607 (2018).

[82] P. Bordia, H. Lüschen, U. Schneider, M. Knap, and I. Bloch, Periodically Driving a Many-Body Localized Quantum System, Nat. Phys. 13, 460 (2017).

[83] N. Goldman, J. Dalibard, M. Aidelsburger, and N. R. Cooper, Periodically Driven Quantum Matter: The Case of Resonant Modulations, Phys. Rev. A 91, 033632 (2015).

[84] A. Haldar, R. Moessner, and A. Das, Onset of Floquet Thermalization, Phys. Rev. B 97, 245122 (2018). 
[85] R. Shankar, Renormalization-Group Approach to Interacting Fermions, Rev. Mod. Phys. 66, 129 (1994).

[86] F. Wegner, Flow-Equations for Hamiltonians, Ann. Phys. (Berlin) 506, 77 (1994).

[87] S. Kehrein, The Flow Equation Approach to ManyParticle Systems, Springer Tracts in Modern Physics (Springer, Berlin, Heidelberg, 2007).

[88] S. J. Thomson and M. Schiró, Time Evolution of ManyBody Localized Systems with the Flow Equation Approach, Phys. Rev. B 97, 060201(R) (2018).

[89] A. Verdeny, A. Mielke, and F. Mintert, Accurate Effective Hamiltonians via Unitary Flow in Floquet Space, Phys. Rev. Lett. 111, 175301 (2013).

[90] H. Sambe, Steady States and Quasienergies of a QuantumMechanical System in an Oscillating Field, Phys. Rev. A 7, 2203 (1973).

[91] T. Giamarchi, Quantum Physics in One Dimension, International Series of Monographs on Physics (Oxford University, New York, 2004).

[92] K. Nomura and K. Okamoto, Phase Diagram of $S=1 / 2$ Antiferromagnetic XXZ Chain with Next-Nearest-Neighbor Interactions, J. Phys. Soc. Jap. 62, 1123 (1993).

[93] F. D. M. Haldane, Spontaneous Dimerization in the $S=\frac{1}{2}$ Heisenberg Antiferromagnetic Chain with Competing Interactions, Phys. Rev. B 25, 4925 (1982).

[94] C. Tomaras and S. Kehrein, Scaling Approach for the Time-Dependent Kondo Model, Europhys. Lett. 93, 47011 (2011).
[95] M. Bukov, L. D’Alessio, and A. Polkovnikov, Universal High-Frequency Behavior of Periodically Driven Systems: From Dynamical Stabilization to Floquet Engineering, Adv. Phys. 64, 139 (2015).

[96] G. Birkhoff and G. Rota, Ordinary Differential Equations (Wiley, New York, 1989), ISBN 9780471860037.

[97] M. Rodriguez-Vega, M. Lentz, and B. Seradjeh, Floquet Perturbation Theory: Formalism and Application to Low-Frequency Limit, New J. Phys. 20, 093022 (2018).

[98] M. Vogl, P. Laurell, A. D. Barr, and G. A. Fiete, Analogue of Hamilton-Jacobi Theory for the Time-Evolution Operator, arXiv:1902.07237.

[99] P. Weinberg and M. Bukov, QuSpin: A Python Package for Dynamics and Exact Diagonalisation of Quantum Many Body Systems Part I: Spin Chains, SciPost Phys. 2, 003 (2017).

[100] A. W. Sandvik, Computational Studies of Quantum Spin Systems, AIP Conf. Proc. 1297, 135 (2010).

[101] C. Zhang, F. Pollmann, S. L. Sondhi, and R. Moessner, Density-Matrix Renormalization Group Study of ManyBody Localization in Floquet Eigenstates, Ann. Phys. (Berlin) 529, 1600294 (2017).

[102] H. De Raedt and B. De Raedt, Applications of the Generalized Trotter Formula, Phys. Rev. A 28, 3575 (1983).

[103] E. Polizzi, Density-Matrix-Based Algorithm for Solving Eigenvalue Problems, Phys. Rev. B 79, 115112 (2009). 